\title{
Mechanisms for tropical upwelling in the stratosphere
}

Article

Published Version

Semeniuk, K. and Shepherd, T. G. (2001) Mechanisms for tropical upwelling in the stratosphere. Journal of the Atmospheric Sciences, 58 (21). pp. 3097-3115. ISSN 15200469 doi: https://doi.org/10.1175/15200469(2001)058<3097:MFTUIT>2.0.CO;2 Available at https://centaur.reading.ac.uk/32845/

It is advisable to refer to the publisher's version if you intend to cite from the work. See Guidance on citing.

Published version at: http://dx.doi.org/10.1175/1520-0469(2001)058<3097:MFTUIT>2.0.CO;2

To link to this article DOI: http://dx.doi.org/10.1175/1520-

0469(2001)058<3097:MFTUIT>2.0.CO;2

Publisher: American Meteorological Society

All outputs in CentAUR are protected by Intellectual Property Rights law, including copyright law. Copyright and IPR is retained by the creators or other copyright holders. Terms and conditions for use of this material are defined in the End User Agreement.

\section{www.reading.ac.uk/centaur}

\section{CentAUR}


Central Archive at the University of Reading

Reading's research outputs online 


\title{
Mechanisms for Tropical Upwelling in the Stratosphere
}

\author{
Kirill Semeniuk And Theodore G. ShePherd \\ Department of Physics, University of Toronto, Toronto, Ontario, Canada
}

(Manuscript received 12 May 2000, in final form 19 April 2001)

\begin{abstract}
The dynamics of the tropical upwelling branch of the stratospheric Brewer-Dobson circulation are examined, with a particular focus on the role of the middle-atmosphere Hadley circulation. Upwelling is examined in terms of both the diabatic circulation and Lagrangian trajectories using a zonally symmetric balance model. The behavior of the wave-driven circulation in the presence of angular momentum redistribution by the Hadley circulation is also considered. The results of the zonally symmetric model are compared with fields from a middle-atmosphere GCM. It is found that the Hadley circulation makes a significant contribution to annual mean tropical upwelling at the upwelling maximum in the vicinity of the stratopause, and can account for most of the annual mean upwelling seen in the GCM. In the mid- to lower stratosphere, the role of the Hadley circulation is much weaker and wave drag appears to be required to explain the observed upwelling, although the Hadley circulation makes a nonnegligible contribution to the annual cycle of the upwelling. Subtropical wave drag can produce annual mean upwelling through a nonlinear mechanism; viscosity is not required. However, the magnitude of the observed upwelling suggests that wave drag must penetrate quite close to the equator.
\end{abstract}

\section{Introduction}

Interest in stratospheric climate and the role played by transport of chemically and radiatively active trace gases and aerosols has brought attention to the dynamics of the Brewer-Dobson circulation in the Tropics. Tropospheric air parcels that enter the stratosphere through the tropical tropopause are most likely to reach high altitudes and have long residence times. This transport pattern reflects the structure of the Brewer-Dobson circulation, which involves diabatic ascent at low latitudes and diabatic descent at higher latitudes. The prevailing view is that upwelling in the lower tropical stratosphere is a consequence of the diabatic circulation induced by planetary wave drag in the extratropics (Holton et al. 1995). The diabatic circulation is also argued to be the agent by which extratropical wave drag influences tropical tropopause temperatures (Yulaeva et al. 1994), leading to a seasonal modulation of water vapor concentration in the upwelling region (Mote et al. 1996).

The leading-order behavior of the diabatic circulation in the extratropics is captured by the linear (or quasigeostrophic) transformed Eulerian mean formulation. However, in the linear system the diabatic circulation produced by extratropical wave drag cannot explain either the observationally inferred location of the upwelling maximum or annual mean tropical upwelling.

Corresponding author address: Dr. Theodore G. Shepherd, Dept. of Physics, University of Toronto, 60 St. George Street, Toronto, ON M5S 1A7, Canada.

E-mail: tgs@atmosp.physics.utoronto.ca
In particular, wave drag confined to the extratropics of one hemisphere, as is the case for the bulk of stratospheric wave drag during solstice seasons, is unable to produce a circulation with upwelling maximizing in the opposite hemisphere as is observed to be the case. This was pointed out by Plumb and Eluszkiewicz (1999) using zonal-mean model experiments, which indicate that the upwelling maximum forms at the low-latitude edge of the wave-drag region. Moreover, without nonlinearity, viscosity and interannual variability, wave drag confined to the extratropics cannot produce an annual mean circulation in the Tropics. This follows from the fact that the annually averaged linear zonal-mean equations have the same form as in steady state, so that the downward control principle of Haynes et al. (1991) applies. Yet, observations of water vapor and methane (Mote et al. 1996) point to persistent tropical upwelling.

The studies of Plumb and Eluszkiewicz (1999) and Sankey (1998) concluded that some form of tropical wave drag (taking the form of viscosity) is necessary to produce annual mean upwelling in the lower tropical stratosphere where nonlinearity is weak. Plumb and Eluszkiewicz (1999) used a linear zonal-mean model with Rayleigh damping to represent the effect of angular momentum forcing by tropical waves, and found that even very weak damping rates can lead to significant annual mean tropical upwelling. Moreover, Sankey (1998) found that including nonlinearity in the viscous model tends to have a suppressing effect on tropical upwelling. This stems from the poleward deformation of the wave-driven diabatic circulation as it advects ab- 
solute angular momentum isopleths away from the equator. However, both studies used models with a uniform radiative equilibrium temperature, which excluded the representation of the middle-atmosphere Hadley circulation (Dunkerton 1989).

More recently, Tung and Kinnersley (2001, hereafter TK) have argued that a significant penetration of the wave-drag-induced diabatic circulation into the opposite hemisphere can occur in the upper stratosphere. They point out that the annual mean downward control argument presented above is flawed since it implicitly assumes that absolute angular momentum isopleths are latitudinally stratified everywhere. In fact, there is a region of significant isopleth deformation in the upper tropical stratosphere and lower mesosphere. As a result, upper-stratosphere wave drag in the winter hemisphere may be important for upwelling in the summer hemisphere subtropics of the lower stratosphere. Such "sideways-downward" control was also noted by Dunkerton (1991). However, upwelling rapidly attenuates below the level of wave drag so that the influence of upperstratospheric wave drag on the lower tropical stratosphere is limited. Certainly the annual mean downward control restriction is valid for the influence of extratropical wave drag at lower levels of the stratosphere.

Plumb and Eluszkiewicz (1999) also pointed out that the Hadley circulation described by Dunkerton (1989) can affect the location of the upwelling maximum. In this paper we investigate further the role of the Hadley circulation in annual mean tropical upwelling. Upwelling is examined in terms of both the diabatic circulation and Lagrangian trajectories. The behavior of the wavedriven circulation in the Tropics in the presence of angular momentum redistribution by the Hadley circulation is also considered. The effect noted by TK will be shown to be closely linked to the action of the Hadley circulation on the state of the upper tropical stratosphere.

We also investigate the inviscid weakly nonlinear regime in the zonal-mean dynamics of the lower tropical stratosphere. Considering the fact that viscosity is not a good model of wave drag (Shepherd et al. 1996), an inviscid mechanism for annual mean upwelling is worth considering. In this light, the work of Plumb and Eluszkiewicz (1999) is revisited to consider the effect of weak nonlinearity in the case of a nearly inviscid balance model.

The numerical results presented here are based on a zonally symmetric nonlinear balance model described in the companion paper (Semeniuk and Shepherd 2001, hereafter referred to as SS); some details are given in section 2. Section 3 deals with the annual mean upwelling produced by the Hadley circulation. The nonlinear mechanism for wave-driven upwelling is considered in section 4 . The effect of angular momentum redistribution by the Hadley circulation on the wave-driven circulation is treated in section 5. In section 6 the idealized model results are compared to simulations from the Canadian Middle Atmosphere Model (CMAM).

\section{Model}

The governing equations which form the basis of the numerical model used for this study are the transformed Eulerian mean (TEM) system (e.g., Andrews et al. 1987)

$$
\begin{array}{r}
\frac{\partial \bar{u}}{\partial t}+\bar{v}^{*}\left(\frac{1}{a} \frac{\partial \bar{u}}{\partial \phi}-f-\bar{u} \frac{\tan \phi}{a}\right)+\bar{w}^{*} \frac{\partial \bar{u}}{\partial z}=\mathcal{F}, \\
\frac{\partial \bar{T}}{\partial t}+\bar{v}^{*} \frac{1}{a} \frac{\partial \bar{T}}{\partial \phi}+\bar{w}^{*} S=Q, \\
\left(f+2 \bar{u} \frac{\tan \phi}{a}\right) \frac{\partial \bar{u}}{\partial z}=-\frac{R}{a H} \frac{\partial \bar{T}}{\partial \phi}, \\
\frac{1}{a \cos \phi} \frac{\partial\left(\bar{v}^{*} \cos \phi\right)}{\partial \phi}+\frac{1}{\rho} \frac{\partial\left(\rho \bar{w}^{*}\right)}{\partial z}=0,
\end{array}
$$

where $\bar{u}, \bar{T}$, and $\left(\bar{v}^{*}, \bar{w}^{*}\right)$ represent the zonal wind, temperature, and residual circulation, respectively. The other terms are defined as follows: $a$ is the planetary radius, $f=2 \Omega \sin \phi$ is the Coriolis parameter, $\rho=\rho_{0}$ $\exp (-z / H)$ is the reference density profile with $H=7$ $\mathrm{km}$ the constant density scale height, and $S \equiv \partial \bar{T} / \partial z+$ $(\kappa / H) \bar{T}$ is the static stability parameter. The EliassenPalm flux divergence representing forcing by wave drag is denoted by $\mathcal{F}$. The diabatic heating is denoted by $Q$. Equation (3) represents thermal-wind balance (a combination of gradient-wind and hydrostatic balance), while (4) represents mass continuity.

Mass continuity together with thermal-wind balance allow (1) and (2) to be combined into a single equation for the residual circulation streamfunction

$$
\begin{aligned}
\mathcal{L}[\psi] & \equiv A \frac{\partial^{2} \psi}{\partial \phi^{2}}+B \frac{\partial^{2} \psi}{\partial \phi \partial z}+C \frac{\partial^{2} \psi}{\partial z^{2}}+D \frac{\partial \psi}{\partial \phi}+E \frac{\partial \psi}{\partial z} \\
& =F,
\end{aligned}
$$

where the streamfunction $\psi$ is defined by $\partial \psi / \partial z=-\rho$ $\cos \phi \bar{v}^{*}$ and $\partial \psi / \partial \phi=a \rho \cos \phi \bar{w}^{*}$. The coefficients of the $\psi$ terms are functionals of $\bar{u}$ and $\bar{T}$, and $F$ is a functional of $\bar{u}, \mathcal{F}$ and $Q$ (see appendix A of SS for details).

The operator $\mathcal{L}$ defined in (5) can be mixed elliptic, parabolic, or hyperbolic depending on the local sign of $B^{2}-4 A C$, which is determined by the distributions of $\bar{u}$ and $\bar{T}$. Hyperbolicity implies an inertially unstable state. The balance model does not resolve inertial instability so an inertial adjustment parameterization is used to prevent $\mathcal{L}$ from becoming hyperbolic (see appendix B of SS for details).

The model surface friction scheme is based on the stress condition of Held and Hou (1980) with a drag coefficient of $C=0.005 \mathrm{~s}^{-1}$. Vertical and horizontal diffusion are included in (1) and (2) depending on the 
numerical experiments. The system is solved by updating $\bar{u}$ and $\bar{T}$ using (1) and (2) at every time step based on the solution of (5) using fields from the previous time step. The MUDPACK multigrid solver (created by John C. Adams at the National Center for Atmospheric Research) is used to obtain the solution of (5).

\section{Annual mean tropical upwelling}

A property of the inviscid linear version of the TEM system is the identical form of its annual average and its steady state in the absence of interannual variability in wave drag and radiative forcing. To a first approximation, the lower-stratosphere Tropics are isolated from the bulk of wave drag, which is primarily in the form of breaking and thermal dissipation of planetary waves occurring poleward of about $20^{\circ}$ latitude as suggested by observed aerosol distributions (Grant et al. 1996) and observational inferences of Eliassen-Palm flux divergence (Rosenlof 1995). The annual mean inviscid linear system exhibits downward control and mass streamlines are confined to these latitudes. As a result, annual mean upwelling is precluded in the lower-stratosphere Tropics, if linearity is assumed to hold at all altitudes. During the year, however, the system is not required to reach steady state and the circulation can penetrate deep into the Tropics and across the equator.

This annual mean downward control behavior may be demonstrated as follows. Consider the annual mean, denoted by \langle\rangle , of the linear TEM system driven by annually periodic wave drag $\mathcal{F}$ and radiative equilibrium temperature $\bar{T}_{\text {rad }}$. The zonal wind equation then takes the form

$$
-f\left\langle\overline{\boldsymbol{v}}^{*}\right\rangle=\langle\mathcal{F}\rangle,
$$

since the tendency term averages to zero and there are no correlation terms that would arise from nonlinear advection. The Coriolis parameter $f$ is zero only at the equator, so if $\langle\mathcal{F}\rangle$ is zero in some latitude range then so must be $\left\langle\bar{v}^{*}\right\rangle$. Without viscosity, mechanical forcing is guaranteed to be zero outside the latitudes of wave drag. Then the time-averaged mass continuity equation implies

$$
\frac{1}{\rho} \frac{\partial\left(\rho\left\langle\bar{w}^{*}\right\rangle\right)}{\partial z}=0
$$

in this region since $\partial\left(\left\langle\overline{\boldsymbol{v}}^{*}\right\rangle \cos \phi\right) / \partial \phi=0$. Equation (7) can be solved by integrating toward the surface from $z$ $=\infty$ where $\rho\left\langle\bar{w}^{*}\right\rangle=0$. Hence, $\left\langle\bar{w}^{*}\right\rangle$ is also zero throughout the region. Thus there cannot be a net annual mean upwelling at these latitudes.

As pointed out by TK, the system cannot be assumed to be linear at all levels. In fact, in the upper-stratosphere Tropics the zonal-mean dynamics are highly nonlinear. Thus annual mean upwelling near the equator in the lower tropical stratosphere could be driven by the sub- tropical dynamics at higher levels. However, there is a limitation on the downward influence of wave drag, which stems from the fact that the vertical velocity decays rapidly as one moves down from the drag region. In steady state it falls off as the inverse of density. In the transient regime, which is particularly relevant in the Tropics, the vertical velocity attenuates even faster (Haynes et al. 1991). The results of TK therefore do not contradict the basic idea in the analysis presented above, that the extratropical pump picture has difficulties explaining annual mean upwelling in the lower tropical stratosphere. Indeed, it is noted by TK that upwelling due to subtropical wave drag is negligible at lower altitudes where the degree of linearity is high, which is consistent with Plumb and Eluszkiewicz (1999).

The inviscid linear form of the balance TEM system supports a Hadley circulation. As in the nonlinear system, it is produced by the incompatibility of the meridional profile of the radiative equilibrium temperature $\bar{T}_{\text {rad }}$ in the Tropics and the gradient-wind balance constraint, given the available angular momentum (Held and Hou 1980; Dunkerton 1989). However, in the linear system the absolute angular momentum $\bar{m}$ distribution is fixed and hence not materially conserved. As a result, the Hadley circulation leads to persistent acceleration of the zonal wind and steady state cannot be reached. Thus, in order for the linear system to have no interannual tendency requires restrictions on the meridional profile of $\bar{T}_{\text {rad }}$. In particular $\bar{T}_{\text {rad }}$ can have no curvature near the equator in the annual mean. In this case a Hadley circulation of the Held and Hou (1980) type is excluded.

A solstitial Hadley circulation (Dunkerton 1989) forced by annually periodic equatorial solar heating gradients can be accommodated by the linear system since the diabatic heating averages to zero in the annual mean. However, the linear solstitial Hadley circulation, which is independent of the wave-driven circulation, cannot produce annual mean upwelling. This can be seen by setting $\langle\mathcal{F}\rangle=0$ in (6). Hence, the wave-driven circulation and the Hadley circulation can contribute to annual mean upwelling in the Tropics only through nonlinearity or viscosity (the latter being a proxy for tropical wave drag).

\section{a. Role of viscosity in annual mean tropical upwelling}

The behavior of the diabatic circulation in the linear TEM system is modified by viscosity. Viscosity is a relaxational mechanical forcing that responds to the zonal wind changes produced by the diabatic circulation or wave drag. In the case of the wave-driven circulation the effect is to allow streamlines to cross $\bar{m}$ contours in steady state. Streamlines can then extend outside the latitudes of the wave drag in both steady state and the annual mean. Viscosity also enables the linear Hadley 
a

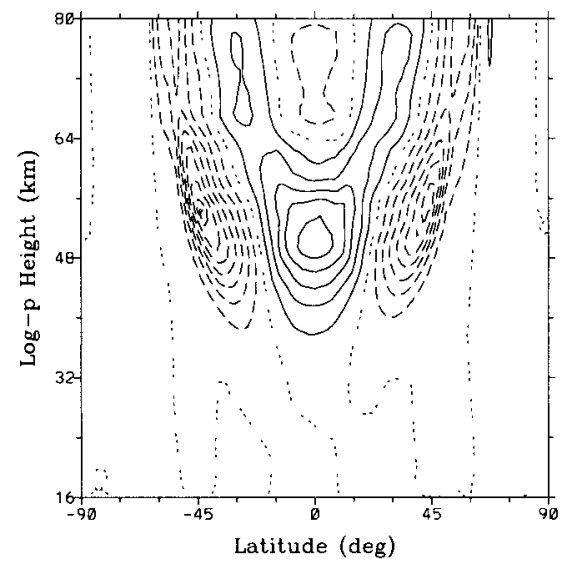

b

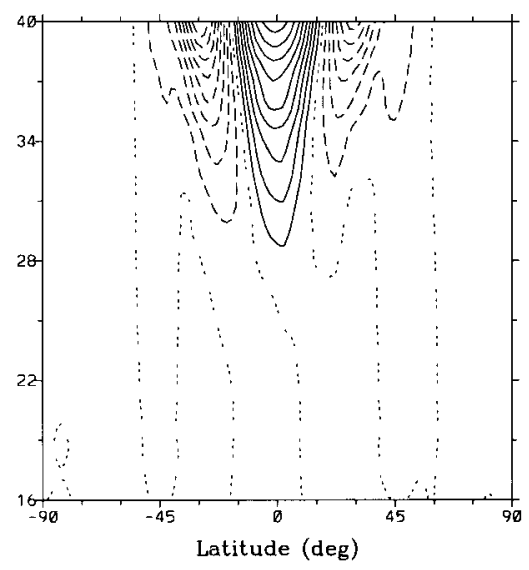

FIG. 1. Annual mean vertical velocity for the balance model simulation without wave drag (average over year 3). (a) Upper stratosphere and mesosphere $\left(2 \times 10^{-4} \mathrm{~m} \mathrm{~s}^{-1}\right)$. (b) Lower stratosphere $\left(3 \times 10^{-5} \mathrm{~m} \mathrm{~s}^{-1}\right)$.

circulation to have a steady state associated with it, although the approachability of this state during solstice seasons depends on the value of the viscosity coefficient.

For the Hadley circulation to evolve to steady state requires either $\bar{m}$ advection or some form of relaxational mechanical forcing to permit flow across fixed isopleths of $\bar{m}$. However, the steady state for the linear viscous system does not resemble that reached in the nonlinear system (Dunkerton 1989, Fig. 19), especially in the upper stratosphere. So nonlinearity is an essential element of the dynamics in these regions.

It was shown by Plumb and Eluszkiewicz (1999) that a significant annual mean tropical upwelling can arise from the wave-driven circulation in the presence of weak viscosity, provided the wave breaking region extends close enough to the equator. If the forcing falls within an equatorial "viscous boundary layer," then the spreading of streamlines of the force-induced circulation across the Tropics is significantly enhanced. The width of this boundary layer is given by $L_{R} P^{1 / 4}$, where $L_{R}$ is the equatorial Rossby radius and $P$ is the ratio of radiative to viscous relaxation times. This effect is linked to parabolic behavior of the streamfunction operator at the equator (see section 5).

Viscosity allows the artificial restriction on the equatorially symmetric part of $\bar{T}_{\text {rad }}$ in the linear system to be removed. The associated component of the Hadley circulation (Held and Hou 1980) can then produce annual mean upwelling. In contrast, the component of the Hadley circulation driven by the equatorially antisymmetric part of $\bar{T}_{\text {rad }}$ which dominates during solstice seasons makes no contribution to annual mean upwelling. This follows from the annual average of Eq. (9) of Plumb and Eluszkiewicz (1999) when the wave drag $D$ and meridional gradient of $\bar{T}_{\text {rad }}$, proportional to $\theta_{\mu}$, are set to zero (the equatorially symmetric component of $\bar{T}_{\text {rad }}$ is assumed to be constant in latitude). Whereas wave drag leaves an annual mean signature in the linear vis- cous system, the diabatic heating associated with this component of the Hadley circulation does not. The mechanical forcing produced by viscosity reflects this. Hence, nonlinearity is required for the solstitial Hadley circulation to drive annual mean upwelling.

\section{b. Tropical upwelling via the Hadley circulation}

The tendency for streamlines and $\bar{m}$ isopleths to deform together in the nonlinear system creates the conditions for annual mean tropical upwelling. In the case of the solstitial Hadley circulation (see SS Fig. 8a), this tendency leads to a deflection of the streamlines away from the equator into the winter hemisphere. As a result there is an asymmetry in the streamfunction $\psi$ that does not average out in the annual mean. The contribution to the annual average from the component of the Hadley circulation associated with the equatorially symmetric part of $\bar{T}_{\text {rad }}$ is an order of magnitude smaller, as discussed further below.

The annually averaged residual vertical velocity $\bar{w}^{*}$ distribution from a simulation using the zonally symmetric balance model without wave drag but with a comprehensive radiative transfer scheme is given in Fig. 1a. There is a region of upwelling maximizing near the stratopause that is flanked by regions of downwelling. Most of the upwelling occurs in the mid- to upper stratosphere where the advection of $\bar{m}$ is greatest due to the large cross-equatorial flow. This feature also explains the increasing latitudinal span of the region of net upwelling with height.

As noted by previous studies, there is a separation of the dynamics between the upper and lower tropical stratosphere. The region of annual mean upwelling almost disappears below approximately $27 \mathrm{~km}$ (see blowup in Fig. 1b) indicating a transition to a predominantly linear regime. There is a change in the latitudinal structure of the upwelling at this level, which argues against 
simple density attenuation as the explanation for the decrease. The transition is the result of the decrease in solar heating gradients at these levels. The behavior in the lower tropical stratosphere is also complicated by the weak component of the Hadley circulation associated with the meridional curvature of $\bar{T}_{\text {rad }}$, which becomes relatively more important at these altitudes in the annual mean. In the lower tropical stratosphere, this component of the Hadley circulation develops a $\bar{w}^{*}$ distribution consisting of a region of weak upwelling overlying a region of weak downwelling (see SS Fig. 3b).

There is some sensitivity of the annual mean upwelling distribution to the choice of diabatic heating scheme. Use of Newtonian relaxation with a uniform damping rate of 0.1 day $^{-1}$ instead of the full radiative transfer scheme leads to the upwelling region penetrating deeper into the lower stratosphere to around $21 \mathrm{~km}$. This results from the enhancement of the diabatic heating distribution at lower levels on account of the relatively greater damping rate (compare Fig. $2 d$ and Fig. $8 \mathrm{~d}$ of SS). However, it is the weak curvature of the climatological $\bar{T}_{\text {rad }}$ at these levels that is the limiting factor on the contribution of the Hadley circulation to annual mean tropical upwelling in the lower stratosphere.

The influence of the Hadley circulation on tropical upwelling was considered by Dunkerton (1997) in a model similar to the one used here but which included realistic prescribed wave drag. Introduction of a small positive curvature into $\bar{T}_{\text {rad }}$ in the lower tropical stratosphere reduced the upwelling by about $50 \%$. The meridional $\bar{T}_{\text {rad }}$ profile was modified in the latitudinal band between $23^{\circ} \mathrm{S}$ and $23^{\circ} \mathrm{N}$, with a reduction of $5 \mathrm{~K}$ at the equator linearly tapering to zero moving away from the equator (T. J. Dunkerton 2000, personal communication). In our model without wave drag, such a $\bar{T}_{\text {rad }}$ curvature modification produced a downwelling with a magnitude about half that of the observationally inferred upwelling, which is consistent with the results of Dunkerton (1997).

A difference is also evident in Fig. 1a between the pattern of upwelling in the mesosphere and stratosphere. Above the stratopause, the single upwelling maximum at the equator splits in two and moves away from the equator. This structure reflects the distribution of the diabatic heating, which develops cooling over the equator in the mesosphere (see SS Fig. 8d). Note that this feature does not appear in the Newtonian cooling simulations.

Figure 2 shows the annual cycle of upwelling at the equator for different altitudes for the same simulation. There is persistent but periodically modulated upwelling near the equator throughout the seasonal cycle in the upper stratosphere. Below about $30 \mathrm{~km}$ the upwelling begins to undergo periodic sign reversals. Peak upwelling occurs earlier in the year at higher levels (e.g., 228 days into the year at $40 \mathrm{~km}$ compared with 257 days at $29 \mathrm{~km}$ ). This can be explained partly by the

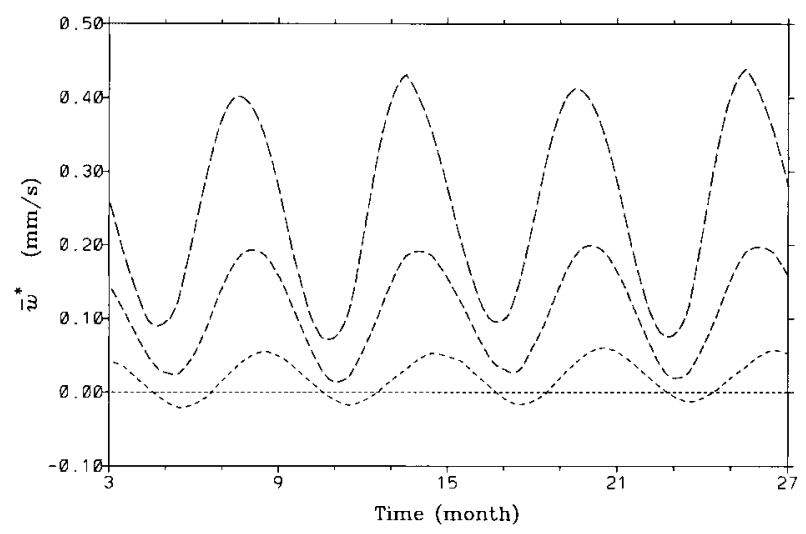

FIG. 2. Balance model vertical velocity at the equator vs time for three different altitudes: 29 (short dash), 35 (medium dash), and 40 $\mathrm{km}$ (long dash).

shortening radiative damping timescale with increasing height, although there is also a lagged penetration of the circulation produced near the heating maximum in the upper stratosphere and lower mesosphere down to lower levels. At lower altitudes the meridional gradient and the meridional curvature of $\bar{T}_{\text {rad }}$ are not large enough to produce a significant Hadley circulation and annual mean upwelling (as indicated by the short dash curve in Fig. 2).

\section{c. Parcel trajectories}

Some sense of the effect of the Hadley circulation on tropical diabatic transport can be obtained from parcel trajectories (in the meridional plane). We use the meridional circulation field from a simulation of the Hadley circulation using the zonal-mean balance model and a Newtonian cooling scheme that relaxes temperatures toward climatological $\bar{T}_{\text {rad }}$ with a uniform damping rate of 0.1 day $^{-1}$ (the first numerical experiment discussed in SS). The integration extends over $3 \mathrm{yr}$ beginning in January.

Parcels initially on the equator move off into the Southern Hemisphere and circulate with a net descent (Fig. 3). The latitudinal span of the circulation and net movement of the parcels off the equator increases with initial parcel altitude. On the other hand, parcels initially located off the equator at $12^{\circ} \mathrm{N}$ oscillate about the equator in latitude with increasing amplitude as they drift upward (Fig. 4). For parcels initially at $12^{\circ} \mathrm{S}$ the behavior is much the same as for those initially on the equator.

The apparent meridional asymmetry in the Hadley circulation indicated by the parcel trajectories in Fig. 3 is a consequence of the initial condition. If the meridional circulation is started from July, then a similar trajectory pattern develops but with initial latitudes mirrored about the equator. The initial-condition-dependent asymmetry is not particular to solstice seasons: parcels initialized during the spring and fall equinoxes also do 


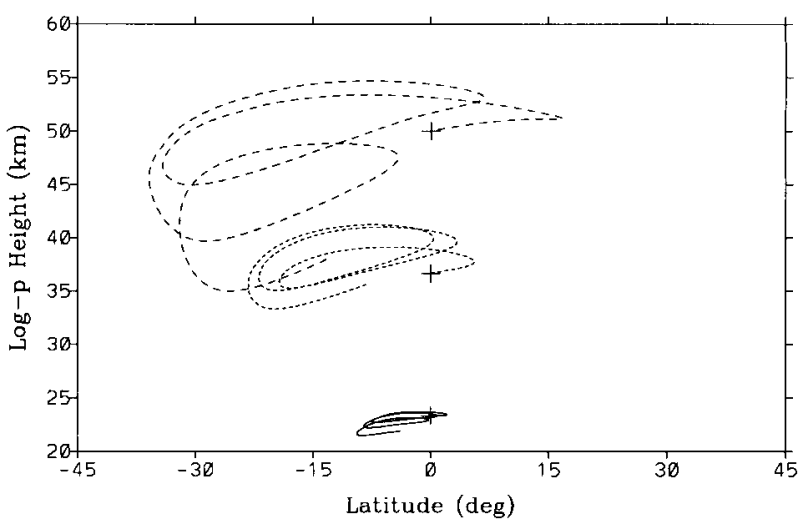

FIG. 3. Parcel trajectories for a 3-yr advection by the balance model residual circulation in the case with no wave drag. Parcels are initially placed on the equator (marked by plus symbol) in the middle of Jan at 23.3 (solid), 36.7 (dash), and $50 \mathrm{~km}$ (long dash).

not behave the same on opposite sides of the equator. This dependence on the start time is one example of the difference between Lagrangian-mean and Eulerianmean quantities.

For parcels with initial positions at latitudes well away from the equator (toward the subtropics) in the middle stratosphere, the overall tendency is less net upward drift and recirculation. This is due to the decreasing mobility of $\bar{m}$ contours at higher latitudes, which translates into increasing linearity. At these latitudes, parcels feel a periodic circulation with little or no annual mean flow. The increase with altitude in the latitudinal excursion of parcels from the equator reflects the streamline behavior. The meridional velocity $\bar{v}^{*}$ is large in the upper stratosphere but has no annual mean component at the equator. It is, however, possible for equatorial parcels to enter the midlatitudes during the winter season in the middle stratosphere, effectively extracting them from the "tropical pipe" (Plumb 1996).

From the tendency of parcels to move downward apart from a very narrow latitude range on one side of the equator, it is evident that the wave-driven circulation is required to account for the broad band of observed

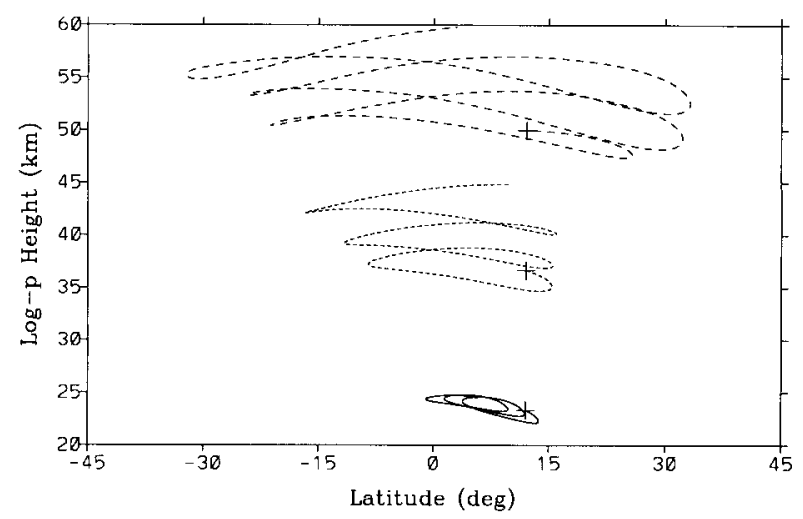

FIG. 4. Same as Fig. 3 but with parcel positions initially at $12^{\circ} \mathrm{N}$.

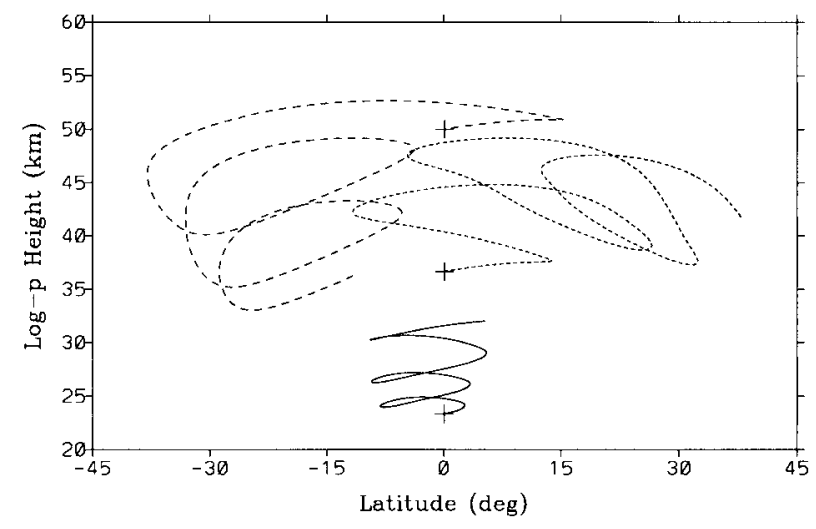

FIG. 5. Same as Fig. 3 but with subtropical wave drag present.

tracer upwelling in the Tropics (Mote et al. 1998). Even though there is a broad region of annual mean Eulerian upwelling due to the Hadley circulation in the upper stratosphere, it is flanked by downwelling regions. As seen by the degree of latitudinal excursion of parcels at these altitudes in Fig. 3, there will therefore be a tendency for parcels to move down away from the equator.

Individual parcel trajectories in the presence of subtropical forcing are presented in Figs. 5 and 6. The initial positions are the same as before. The form of the wave drag is given explicitly in the next section. It has a magnitude of $-1.5 \mathrm{~m} \mathrm{~s}^{-1}$ day $^{-1}$ with the peak value at $35-\mathrm{km}$ altitude and $30^{\circ}$ latitude in both hemispheres. The forcing is applied for half the year overlapping the winter season. The presence of the wave-driven circulation alters the behavior of parcels initially on the equator. In the lower stratosphere there is a significant enhancement of the upward drift. In the middle stratosphere parcels are pulled completely out of the Tropics into the winter hemisphere. There is little effect in the upper stratosphere since the forcing distribution used does not produce a significant circulation there. In reality, there is a great deal of subtropical forcing at these levels, which will have a significant impact.

For parcels starting at $12^{\circ} \mathrm{N}$ the drift toward the Pole

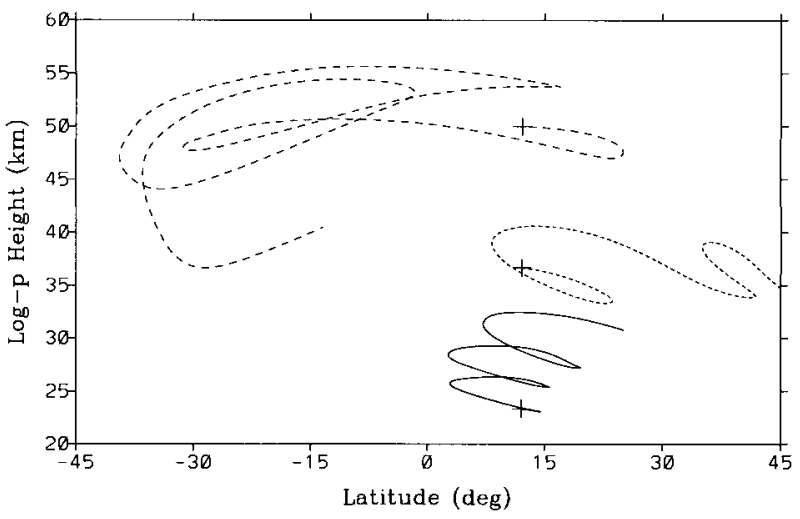

FIG. 6. Same as Fig. 4 but with subtropical wave drag present. 
is clearly evident except for the parcel in the upper stratosphere that is put on a trajectory downward in the summer hemisphere. Once again, this is due to the weakness of the modeled wave-driven circulation at these levels, but indicates that parcel trajectories are sensitive to relatively small path alterations. It also points to the requirement for a realistic wave drag distribution to produce the correct transport behavior.

\section{Tropical upwelling via the wave-driven circulation}

The limited contribution of the Hadley circulation to annual mean upwelling in the lower tropical stratosphere necessitates consideration of other mechanisms. A potential mechanism, which is not predicated on viscosity, is nonlinearity in the circulation driven by extratropical wave drag. As pointed out previously, advective nonlinearity enables the system to get around the annual mean downward control limitation, although it is not clear a priori whether it can lead to net upwelling.

In this section we present experiments similar to those of previous studies (Plumb and Eluszkiewicz 1999; Sankey 1998) based on the balance model including some simulations with no prescribed viscosity. As with previous studies, the diabatic heating is approximated by Newtonian cooling (here with a uniform damping rate of $1 / 6$ day $^{-1}$ ) and with the radiative equilibrium temperature set to a constant value of $240 \mathrm{~K}$. This suppresses the formation of the Hadley circulation and inertially unstable regions. Such an approach is partly justified by the weak nonlinearity of the system at lower altitudes, which allows the effect of wave drag to be considered separately to a reasonable approximation (see SS).

The response of the linear and nonlinear systems to an idealized wave drag poleward of $5^{\circ}$ and $25^{\circ}$ from the equator was considered by Sankey (1998). The wave drag used in that study had a simple localized distribution with a $\sin ^{2}$ time dependence with an annual period and a magnitude of $-10 \mathrm{~m} \mathrm{~s}^{-1} \mathrm{day}^{-1}$. For wave drag approaching the equator to within $5^{\circ}$, Sankey found strong upwelling extending deep into the opposite hemisphere, in both the linear and nonlinear versions of the model. The nonlinear system, however, produced a weaker mean upwelling in the Tropics than did the linear system. The stronger annual mean upwelling in the linear system is partly due to the wave drag overlapping the viscous boundary layer described by Plumb and Eluszkiewicz (1999). In the case of the forcing situated poleward of $25^{\circ}$, the region of upwelling was found to diminish significantly and to be confined close to the forcing region both with and without nonlinearity. Sankey did not assess the effect of viscosity on the upwelling produced by the nonlinear model.

It happens that there is a qualitatively different response when the equatorial edge of the forcing region is situated at latitudes intermediate to those considered by Sankey (1998), for example, $15^{\circ}$. To demonstrate this, the balance model is subjected to a simple forcing of the form $\mathcal{F}(\phi, z, t)=F_{\mathrm{o}} Y(\phi) Z(z) H(t)$. The forcing magnitude $F_{\mathrm{o}}$ is $-3 \mathrm{~m} \mathrm{~s}^{-1}$ day $^{-1}$. The latitudinal dependence is defined by

$$
Y(\phi)= \begin{cases}\frac{1}{2}-\frac{1}{2} \cos \left(\pi \frac{\phi-\phi_{1}}{\phi_{2}-\phi_{1}}\right) & \text { for } \phi_{1} \leq \phi \leq \phi_{2} \\ \frac{1}{2}+\frac{1}{2} \cos \left(\pi \frac{\phi-\phi_{2}}{\phi_{3}-\phi_{2}}\right) & \text { for } \phi_{2} \leq \phi \leq \phi_{3} \\ 0 & \text { otherwise }\end{cases}
$$

with $\phi_{1}$ being the subtropical limit of the forcing, $\phi_{2}$ the latitude of its maximum, and $\phi_{3}$ its northern limit. The vertical dependence is defined by

$$
Z(z)= \begin{cases}\frac{1}{2}+\frac{1}{2} \cos \left[2 \pi \frac{z-\frac{1}{2}\left(z_{1}+z_{2}\right)}{z_{2}-z_{1}}\right] & \text { for } z_{1}<z<z_{2} \\ 0 & \text { otherwise }\end{cases}
$$

with $z_{1}=16 \mathrm{~km}$ the lower limit and $z_{2}=50 \mathrm{~km}$ the upper limit of the forcing region. The maximum forcing level is at $33 \mathrm{~km}$, but the results are the same if the forcing is shifted upward or downward (except near the upper and lower boundary). The time dependence is defined by

$$
H(t)= \begin{cases}\frac{1}{2}-\frac{1}{2} \cos \left(\pi \frac{t-t_{1}}{t_{2}-t_{1}}\right) & \text { for } t_{1} \leq t \leq t_{2} \\ 1 & \text { for } t_{2} \leq t \leq t_{3} \\ \frac{1}{2}+\frac{1}{2} \cos \left(\pi \frac{t-t_{3}}{t_{4}-t_{3}}\right) & \text { for } t_{3} \leq t \leq t_{4} \\ 0 & \text { otherwise. }\end{cases}
$$

The forcing ramps up between day $t_{1}=0$ and day $t_{2}=$ 46 , persists, and then diminishes between day $t_{3}=137$ and day $t_{4}=183$. The results should be qualitatively similar for all localized and single-signed subtropical forcing within the same latitudinal limits.

For this simulation the model horizontal diffusion coefficient was set to $500 \mathrm{~m}^{2} \mathrm{~s}^{-1}$ and the vertical diffusion coefficient to $0.3 \mathrm{~m}^{2} \mathrm{~s}^{-1}$. In fact, for weak forcing in the subtropics these coefficients can be set to zero in the zonal-mean balance model simulations with uniform $\bar{T}_{\text {rad }}$. Numerical instability becomes an issue for the model used here only in the presence of inertial instability.

Latitudinal profiles of the annual mean $\bar{w}^{*}$ for the linear and nonlinear cases at $33 \mathrm{~km}$, the level of the forcing maximum, are given in Fig. 7 for $\phi_{1}=15^{\circ}$. At this latitude, the forcing region is not overlapping the 


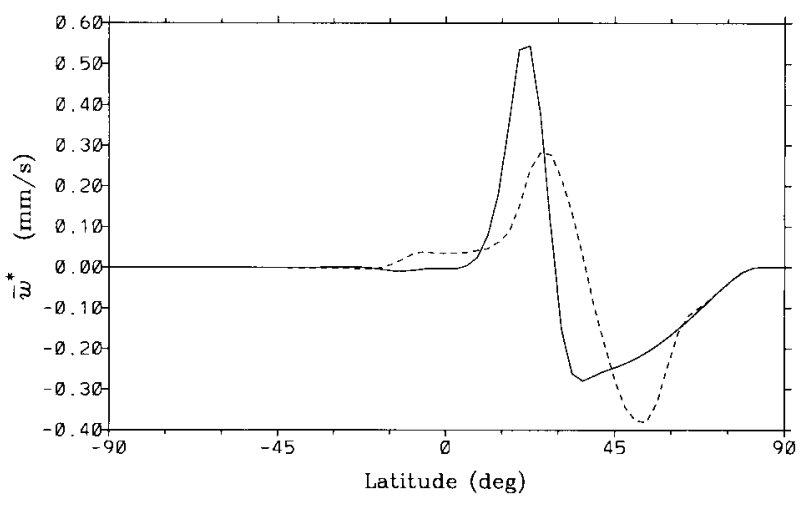

FIG. 7. Meridional profile of the annual mean $\bar{w}^{*}$ at $33 \mathrm{~km}$ in the sixth year of the simulation for the linear (solid) and nonlinear (dash) balance model. Here, $\phi_{1}=15^{\circ}, \phi_{2}=30^{\circ}$, and $\phi_{3}=80^{\circ}$.

viscous equatorial boundary layer described by Plumb and Eluszkiewicz (1999). This can be verified explicitly by making the approximation

$$
\alpha_{u} \bar{u} \sim K_{z z} \frac{\partial^{2} \bar{u}}{\partial z^{2}}+K_{y y} \frac{\partial^{2} \bar{u}}{\partial y^{2}}
$$

so that

$$
\alpha_{u} \sim \frac{K_{z z}}{L_{z}^{2}}+\frac{K_{y y}}{L_{y}^{2}},
$$

where $L_{z}$ and $L_{y}$ are the vertical and meridional scales of $\bar{u}$. For the simulations shown here $K_{z z}=0.3 \mathrm{~m}^{2} \mathrm{~s}^{-1}$, $K_{y y}=500 \mathrm{~m}^{2} \mathrm{~s}^{-1}, L_{z} \approx 10000 \mathrm{~m}$, and $L_{y} \approx 3 \times 10^{5}$ $\mathrm{m}$. (These length scales are derived from consideration of the actual values of diffusion in the model.) So the vertical diffusion dominates and $\alpha_{u} \approx 0.00074 \mathrm{day}^{-1}$, which gives a viscous boundary layer width of $L_{c} \sim$ $\left(\alpha_{u} / \alpha_{T}\right)^{1 / 4} L_{R} \approx 650 \mathrm{~km}$ or $5.8^{\circ}\left(L_{R} \approx 2500 \mathrm{~km}\right)$.

The fact that the forcing lies outside the equatorial boundary layer is also seen by the fact that in the linear case the upwelling region is confined in latitude at the equatorial edge of the forcing region. In the nonlinear case, by contrast, there is a region of weak upwelling penetrating into the opposite hemisphere. The balance of terms in the zonal momentum equation shows that viscosity is not responsible for the formation of this feature. This is in contrast to the situation with $\phi_{1}=$ $5^{\circ}$ when near-equatorial upwelling in the linear case is stronger than in the nonlinear case, in accordance with the results of Sankey (1998).

If we consider the time dependence of $\bar{w}^{*}$ at $33 \mathrm{~km}$ on the equator (Fig. 8), then it is apparent that in the nonlinear case the upwelling phases dominate the downwelling phases. It is also evident that the nonlinear system reaches equilibrium faster than the linear system from the time the periodic forcing is turned on; the linear evolution asymptotes over a much longer period to a situation where the upwelling and downwelling phases have a higher degree of cancellation. In both systems there is still some annual mean transience in the Tropics

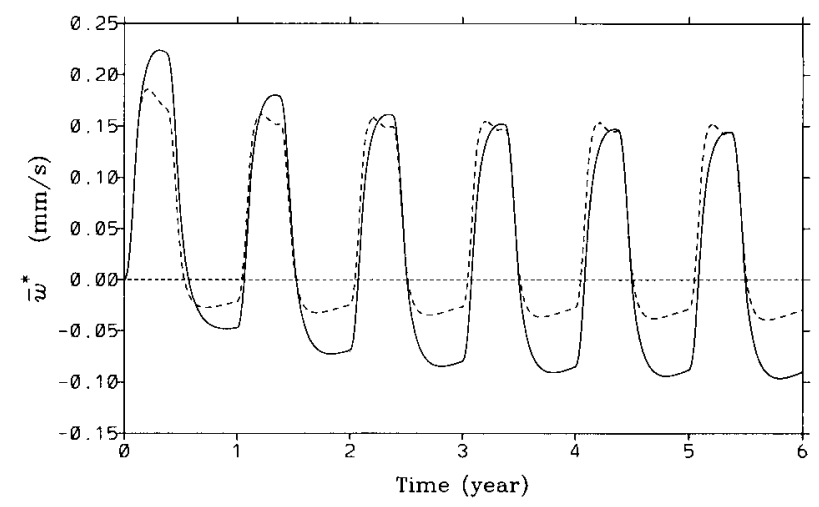

FIG. 8. Time dependence of $\bar{w}^{*}$ at $33 \mathrm{~km}$ on the equator for the simulation of Fig. 7 with the linear model (solid) and nonlinear model (dash).

even after 6 model years, but the balance of terms in the Tropics implies that this transience is not responsible for the upwelling pattern in the nonlinear case.

An important parameter is the radiative damping rate, which has a significant effect on the lifetime of startup transients. Using a much smaller value of $\alpha(0.025$ day $^{-1}$ instead of $0.17 \mathrm{day}^{-1}$ ), more characteristic of the lower tropical stratosphere, results in a much longer transient phase. After the same simulation period of 6 $\mathrm{yr}$ (not shown), the difference in upwelling near the equator between the linear and nonlinear models is very small. In the long-time limit the difference between the two models becomes more apparent. However, this result suggests that transients associated with interannual variability may play a significant role in the real atmosphere.

The net annual mean upwelling in the nonlinear system seen in Fig. 7 points to major differences in the pattern of evolution compared to the linear system. The diabatic heating induced during the active forcing phase produces a downwelling during the inactive phase (the time dependence used here is for clarity; a more slowly varying forcing will still be subject to the same constraint). In the linear system this downwelling has enough amplitude and duration to cancel the upwelling in the annual mean, while in the nonlinear system it does not.

Based on Fig. 9 a certain picture emerges of the asymmetry between the linear and nonlinear response to the applied periodic forcing. The circulation in the nonlinear system deforms $\bar{m}$ surfaces in a manner that leads to a weaker induced temperature anomaly at low latitudes. In particular, near the equatorial edge of the forcing there is a concentration of $\bar{m}$ isopleths in latitude that acts like an enhancement of the Coriolis parameter. This leads to a poleward shift in the streamfunction distribution and a weaker meridional circulation at these latitudes. In the linear system, in contrast, the fixed $\bar{m}$ distribution implies that the circulation is greater at the equatorial edge of the forcing. Hence the induced dia- 

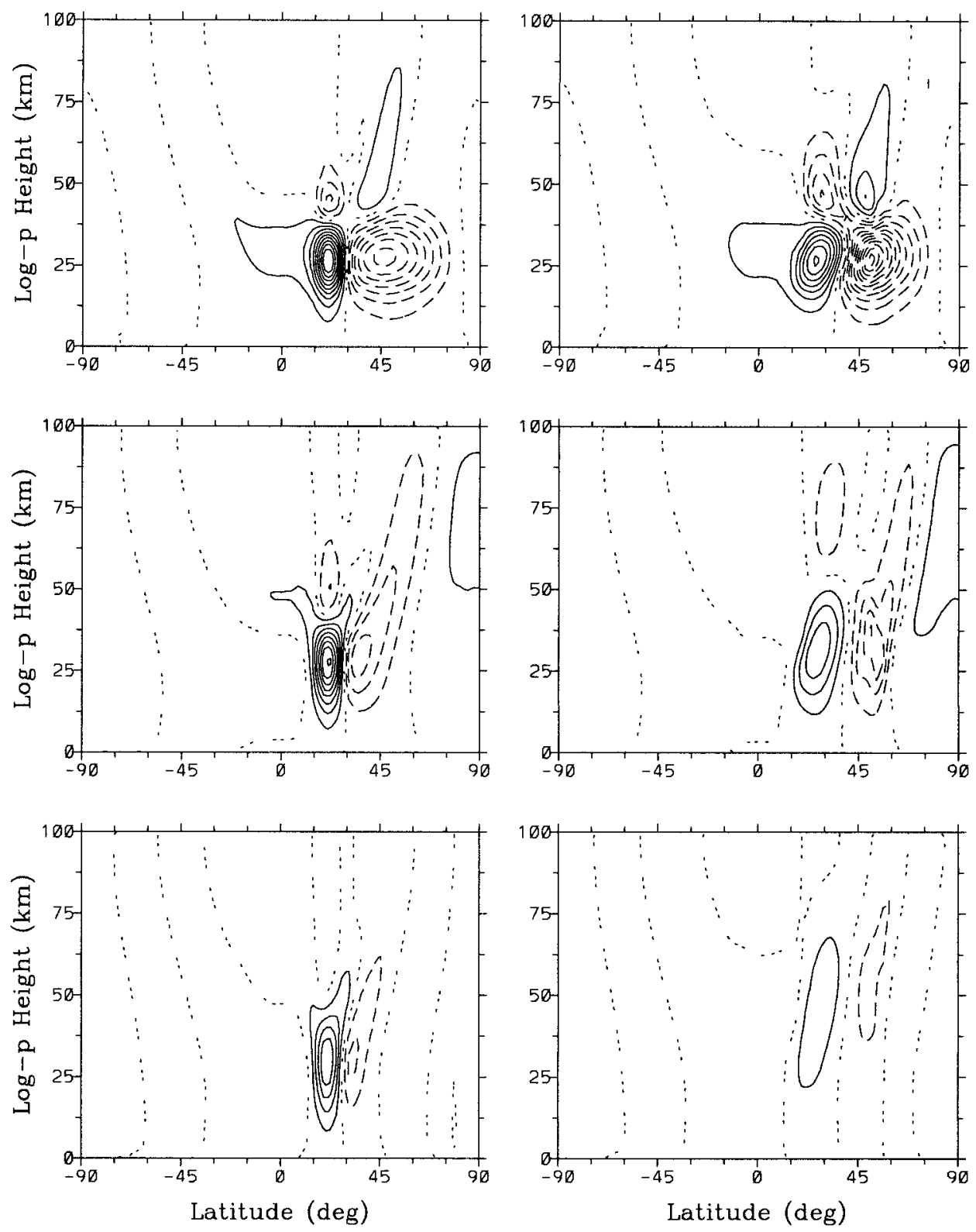

FIG. 9. Diabatic heating at 4 (top), 7 (middle), and 10 months (bottom) for the linear (left panels) and nonlinear model (right panels) for the run of Fig. 7. Note the weakening of the heating in month 10 (during the "off" phase of the forcing) in the nonlinear case. Contour interval used is $0.1 \mathrm{~K}^{\text {day }}{ }^{-1}$ for all panels.

batic heating at low latitudes is larger in the linear than in the nonlinear system.

The weaker induced diabatic heating in the nonlinear system produces a weaker and less persistent $\bar{w}^{*}$ in the Tropics after the forcing is turned off. The increased rate of decay to steady state in the nonlinear system is again linked to the effectively greater Coriolis parameter. The latitudinal concentration of $\bar{m}$ contours lasts for some time as the system relaxes to radiative equilibrium. Given an initial diabatic heating distribution, the rate of evolution to steady state increases as the Coriolis parameter is increased. In fact, the concentra- tion of $\bar{m}$ isopleths also allows the nonlinear system to approach steady state faster when the forcing is applied. In contrast, transience is more persistent in the linear system due to the weak low-latitude Coriolis parameter.

To test whether viscosity was important for the nonlinear system, the diffusion coefficients were reduced to $0.02 \mathrm{~m}^{2} \mathrm{~s}^{-1}$ for the vertical and $5 \mathrm{~m}^{2} \mathrm{~s}^{-1}$ for the horizontal. This had very little effect on the annual mean upwelling profile. So diffusion cannot explain the annual mean upwelling in the nonlinear system (see later).

The above results demonstrate that it is possible for subtropical wave drag no closer than $15^{\circ}$ to the equator 

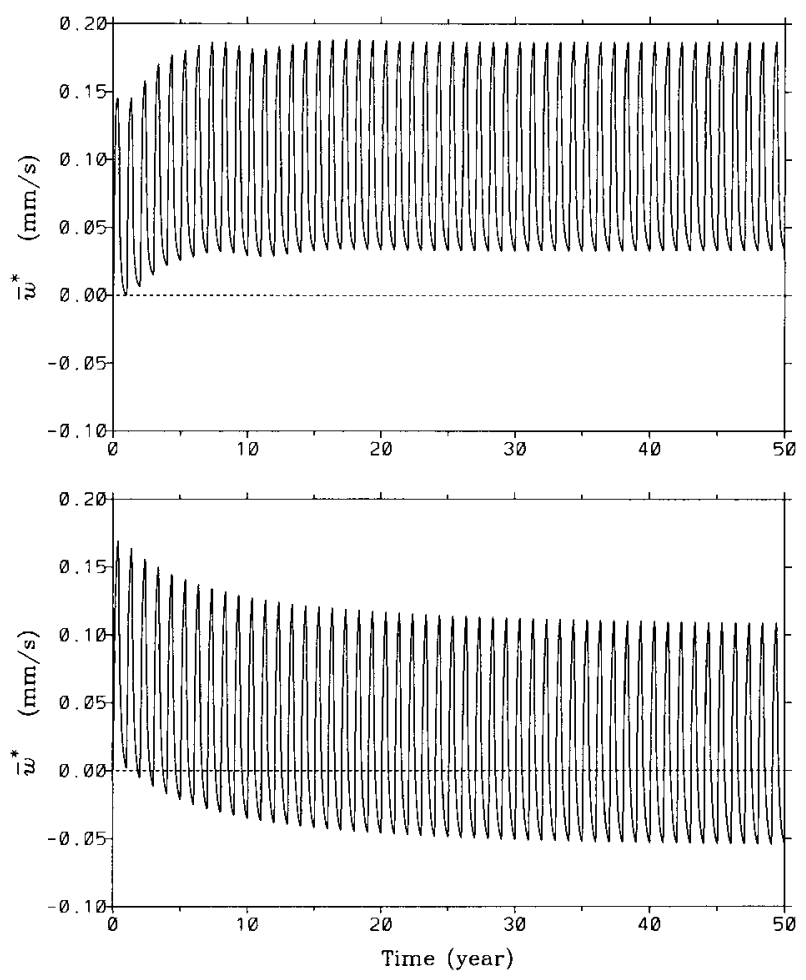

FIG. 10. Time series of $\bar{w}^{*}$ at $33 \mathrm{~km}$ on the equator for the inviscid nonlinear (upper panel) and linear (lower panel) models. The forcing parameters are $\phi_{1}=6^{\circ}, \phi_{2}=30^{\circ}$ and $\phi_{3}=80^{\circ}$. (So the forcing is closer to the equator than in Fig. 7.)

to produce annual mean upwelling through a nonlinear mechanism. However, the upwelling produced in our experiments is too weak to explain the observed values in the lower tropical stratosphere even though the forcing amplitude is larger than typical values for subtropical wave drag (Rosenlof 1995, Fig. 8). The annual mean upwelling obtained at $33 \mathrm{~km}$ is about $10 \%$ of the observationally inferred value at $24 \mathrm{~km}$. A more realistic force distribution may produce a stronger upwelling due to contributions from other levels that are not forced to the same degree in the model. However, for upwelling at a given level, density stratification limits the contribution from higher levels (assuming that any force magnitude increase with height is much slower than exponential - as appears to be generally the case in the atmosphere). On the other hand, the results of TK imply that wave drag in the upper stratosphere low latitudes can affect upwelling in the lower tropical stratosphere. We consider this problem from a different angle in section 5 .

One of the assumptions underlying the work here is that low-latitude wave drag is confined to the surf zone, which at these altitudes is located farther from the equator than it is in the upper stratosphere. While there is strong evidence of the isolation of the lower tropical stratosphere based on tracer observations (Grant et al. 1996), this does not necessarily imply that there is iso-

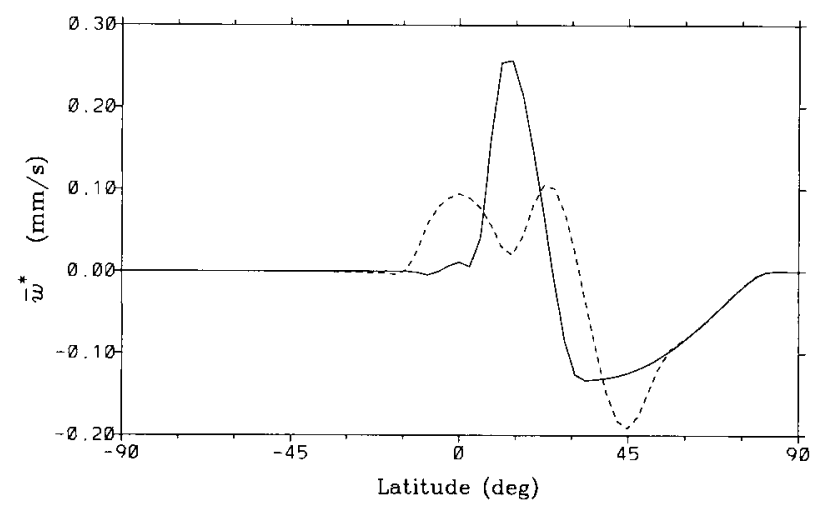

Fig. 11. Annual mean of $\bar{w}^{*}$ on the 33-km level after $50 \mathrm{yr}$ of simulation for the inviscid linear (solid) and nonlinear (dash) models.

lation in terms of wave drag. Planetary waves of extratropical origin that are not breaking and tropical waves can still damp the zonal wind through thermal dissipation. In fact, GCMs such as CMAM do not have the resolved wave drag confined to the surf zone and there is weak Eliassen-Palm flux divergence extending to the equator. However, it should be cautioned that the absence of a sharp surf-zone edge in these models may be a consequence of their coarse resolution.

Therefore, the above experiments were repeated to see what effect a forcing distribution extending closer to the equator and having a more realistic magnitude $\left(-1.5 \mathrm{~m} \mathrm{~s}^{-1}\right.$ day $^{-1}$ at $30^{\circ}$, decaying to zero at $\left.6^{\circ}\right)$ would have on the upwelling in the absence of explicit model diffusion (grid diffusion is unavoidable). The results are given in Figs. 10 and 11 for a simulation lasting 50 model years. The linear model equatorial upwelling shows a monotonic trend to a state with little or no upwelling, whereas the nonlinear model upwelling appears to equilibrate about a positive value reached after less than a third of the integration time. It appears that grid diffusion is playing a minor role, possibly giving a very small annual mean upwelling in the linear model in the long-time limit. The interannual variation in the nonlinear case (Fig. 10) was not sensitive to the radiative damping rate.

A constraint on the possible distribution of the imposed wave drag is the degree of $\bar{m}$ deformation that results. Plumb and Eluszkiewicz (1999) argued that the pattern of $\bar{m}$ isopleths in the lower tropical stratosphere is indicative of a linear regime. Considering the $\bar{m}$ field at the peak deformation by the forcing in the inviscid experiment (Fig. 12), the deflection of isopleths is indeed limited except near the equator. The pattern would at first appear to suggest a higher degree of nonlinearity than is evident in the $\bar{m}$ distribution of CMAM (see SS Fig. 19). However, the comparison is misleading since the contour with the largest value (at this contour interval) closes off near the tropopause in the case of the GCM. The deformation of $\bar{m}$ is evident from its meridional profile in the lower tropical stratosphere in both 


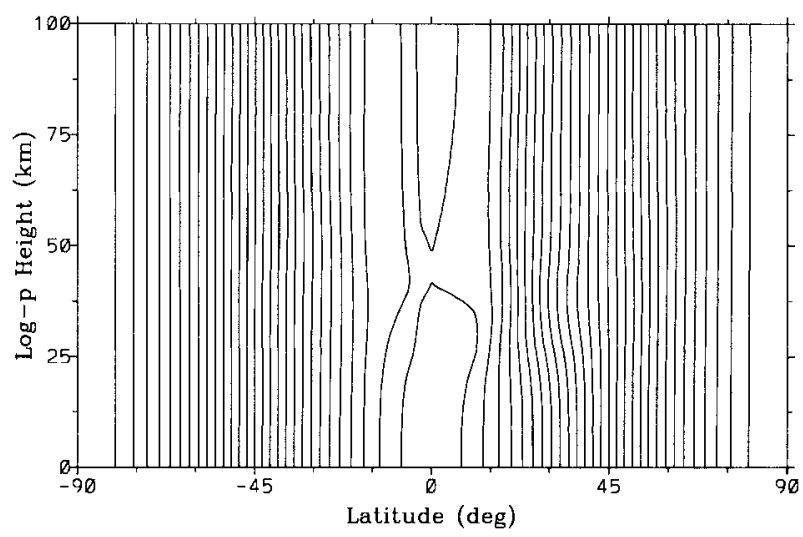

FIG. 12. Distribution of $\bar{m}$ for the inviscid nonlinear model at $t=$ 105 days in year 50 of the simulation.

CMAM and the COSPAR International Reference Atmosphere (CIRA) (Flemming et al. 1990) data (Fig. 13, top panel). Of course the CIRA $\bar{m}$ is not based on direct observations but is derived from the observed temperature field using the thermal wind relation. Nevertheless, the deformation of $\bar{m}$ in the lower tropical stratosphere in both fields is suggestive of weak cross-equatorial advection. It thus appears that weak nonlinearity cannot be excluded from the zonal-mean dynamics of the lower tropical stratosphere on the basis of the $\bar{m}$ distribution.

However, the spatial distribution of the forcing used in the inviscid experiment above still does not produce the right magnitude of annual mean upwelling at the equator $(25 \%$ of the observed value at $24 \mathrm{~km})$. One possible explanation is the unrealistic nature of the $\bar{m}$ distribution in the idealized model with uniform $\bar{T}_{\text {rad }}$, which lacks the equatorial asymmetry of the $\bar{m}$ field in the lower stratosphere found in GCMs and the real atmosphere. As will be shown in the next section, the $\bar{m}$ distribution affects the intensity of tropical upwelling. Another possibility is that wave drag needs to extend to the equator to produce the level of upwelling inferred from observations. Such a hypothesis is supported, for example, by the CMAM Eliassen-Palm flux divergence of resolved waves.

In Figs. 14 and 15 are presented the results of an inviscid model run with the forcing extending to the equator. The upwelling magnitude is almost double that for the previous experiment in the nonlinear case, while the linear model is now producing similar levels of upwelling on the equator. So even without viscosity the behavior of the linear model is very similar to the viscous case in the region of weak forcing near the equator. From Fig. 15 it is clear that this is not a transient effect in the linear model, except at latitudes where the forcing does not reach, where there is an indication of a slow decay in the annual mean upwelling with time.

The results of this section support the conclusion of Plumb and Eluszkiewicz (1999) that tropical wave drag is necessary to produce realistic levels of upwelling. However, the nonlinear mechanism considered here tak-
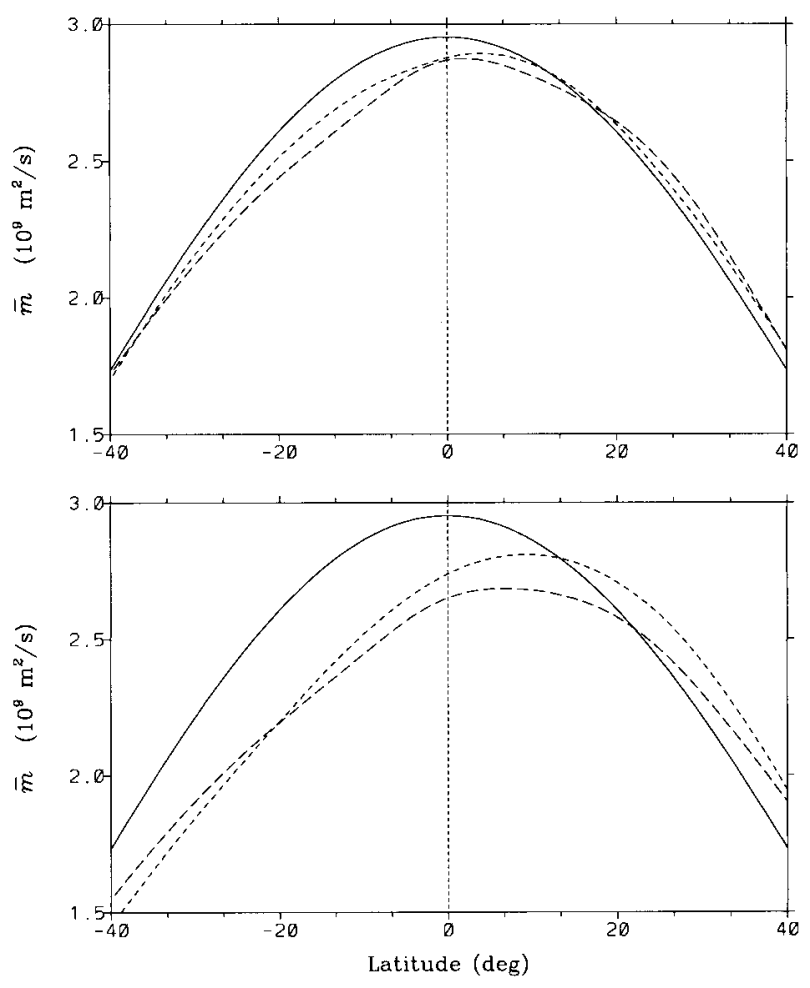

FIG. 13. Meridional profiles of $\bar{m}$ at $25 \mathrm{~km}$ (upper panel) and 50 $\mathrm{km}$ (lower panel) based on Jan zonal wind fields from CIRA (short dash) and from CMAM (long dash). A profile corresponding to no zonal flow is given for reference (solid curve).

en together with the effect described by TK imply that the magnitude of wave drag in the lower tropical stratosphere does not have to be as large as required by the linear model.

\section{Impact of the Hadley circulation on the wave- driven circulation in the Tropics}

The streamfunction operator for the balance TEM system can become nonelliptic at the equator and adjacent regions depending on the zonal wind distribution. This has implications for the behavior of the wave-driven

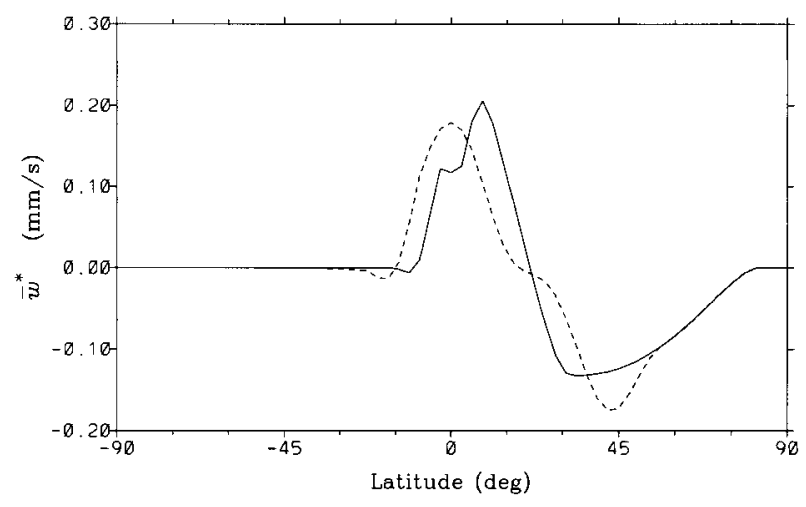

FIG. 14. Same as Fig. 11 but for $\phi_{1}=0$. 

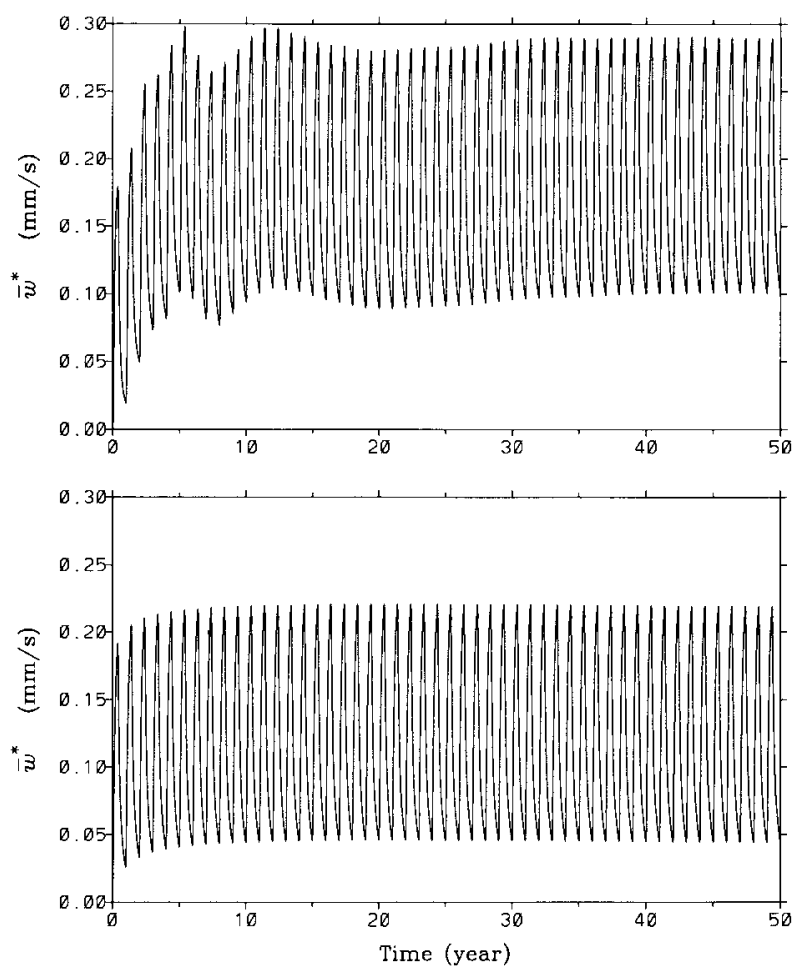

FIG. 15. Same as Fig. 10 but for $\phi_{1}=0$.

circulation in the Tropics. Meridional advection by the Hadley circulation moves the $\bar{m}$ maximum off the equator into the winter hemisphere, causing the system to become inertially unstable. The resulting inertial adjustment modifies the zonal flow and temperature in a manner that keeps the system parabolic. As discussed in SS, the deformation is most pronounced in the upper stratosphere and lower mesosphere where cross-equatorial advection and meridional $\bar{T}_{\text {rad }}$ gradients are large. The impact of $\bar{m}$ and $\bar{T}$ deformations on the wave-driven circulation is the focus of this section.

We consider the influence of the variable properties of the streamfunction operator $\mathcal{L}$ on the wave-driven circulation in a linearized version of the model described in SS forced with a switch-on wave drag $\mathcal{F}$. This approach captures the essence of the instantaneous response in the nonlinear system. Thermal-wind balance leads to the absence of self-advection in the TEM system. This implies that the wave-driven circulation only sees the distribution of $\bar{m}$ and $\bar{T}$, which can be regarded as given. However, the linearized model fails to respond to the feedback of changes in $\bar{m}$ and $\bar{T}$ on the evolution of the system so it will differ from the nonlinear one when used prognostically.

Parabolicity in $\mathcal{L}$ spanning a range of latitudes can produce the behavior of the wave-driven circulation seen near the equator but at higher latitudes. To demonstrate this, we consider two highly idealized baratropic basic states (shown in Fig. 16): one with a broad parabolic region in the Tropics, and one that corresponds

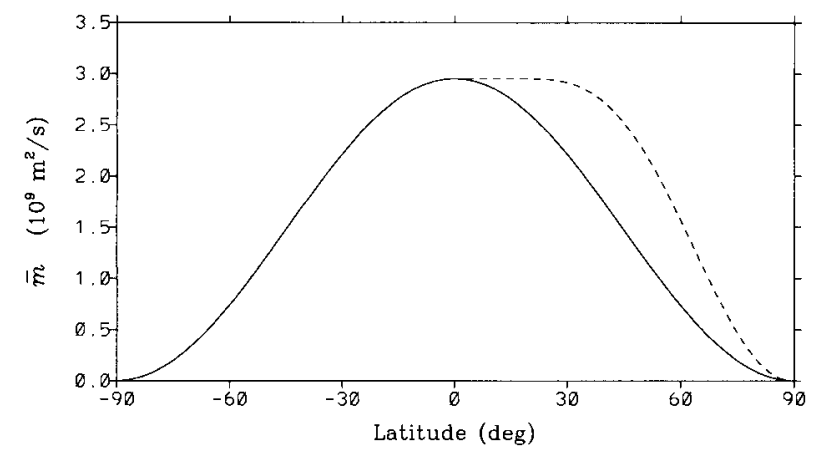

FIG. 16. Profiles in latitude of the reference state $\bar{m}$ for the two cases used to test parabolic streamfunction behavior: no zonal wind or strong $\bar{m}_{\phi}$ (solid) and parabolic or homogenized $\bar{m}$ (dash).

to no zonal flow (or strong ellipticity). The streamfunction and vertical velocity in each of the cases is presented in Figs. 17 and 18 for switch-on forcing of the form

$$
\mathcal{F}(\phi, z)=F_{\mathrm{o}} \exp \left[-\left(\frac{\phi-\phi_{\mathrm{o}}}{10^{\circ}}\right)^{2}-\left(\frac{z-40 \mathrm{~km}}{10 \mathrm{~km}}\right)^{2}\right],
$$

with $F_{\mathrm{o}}=-2 \times 10^{-5} \mathrm{~m} \mathrm{~s}^{-2}$, centered at $\phi_{\mathrm{o}}=45^{\circ} \mathrm{N}$, and in Figs. 19 and 20 for the same forcing centered at $\phi_{\mathrm{o}}=30^{\circ} \mathrm{N}$.

When the region of parabolicity extends into the subtropics, both the instantaneous and long-term streamfunction penetrate farther away from the forcing region and intensify (compare the upper and lower panels of Fig. 17). The distribution of tropical upwelling is no longer confined to the subtropics but extends well into the opposite hemisphere and has no latitudinal gradient in the parabolic region (lower right panel, Fig. 18). The latitudinal confinement by $\bar{m}$ isopleths breaks down in the parabolic $(\partial \bar{m} / \partial \phi=0)$ region.

A similar effect occurs when $\mathcal{F}$ is moved to lower latitudes (cf. Fig. 19 and Fig. 17), except that in this case there is less latitudinal confinement of streamlines and an intensification of the circulation in the strongly elliptic case as well. This reflects the weakening of $\partial \bar{m} / \partial \phi$ with decreasing latitude and the increasing parabolicity of $\mathcal{L}$. In the strongly parabolic case the impact of shifting the forcing region is much larger. Compared to the $\phi_{\mathrm{o}}=45^{\circ} \mathrm{N}$ result, the vertical velocity increases by six times in the Tropics at day 100 (lower right panel, Fig. 20). In contrast, the change in upwelling in the strongly elliptic case is small (upper right panel, Fig. 20).

The expression for $\mathcal{L}$ linearized around these heightindependent basic states simplifies (5) to

$$
\frac{A}{\cos \phi} \frac{\partial}{\partial \phi}\left(\frac{1}{\cos \phi} \frac{\partial \psi}{\partial \phi}\right)+C \rho \frac{\partial}{\partial z}\left(\frac{1}{\rho} \frac{\partial \psi}{\partial z}\right)=F
$$

[A, $C$, and $F$ are the same as in appendix B of SS but with terms involving the vertical derivative of $M \equiv$ $(\bar{m} / a)^{2}$ set to zero.] If we take $\Delta z$ to be the vertical scale 

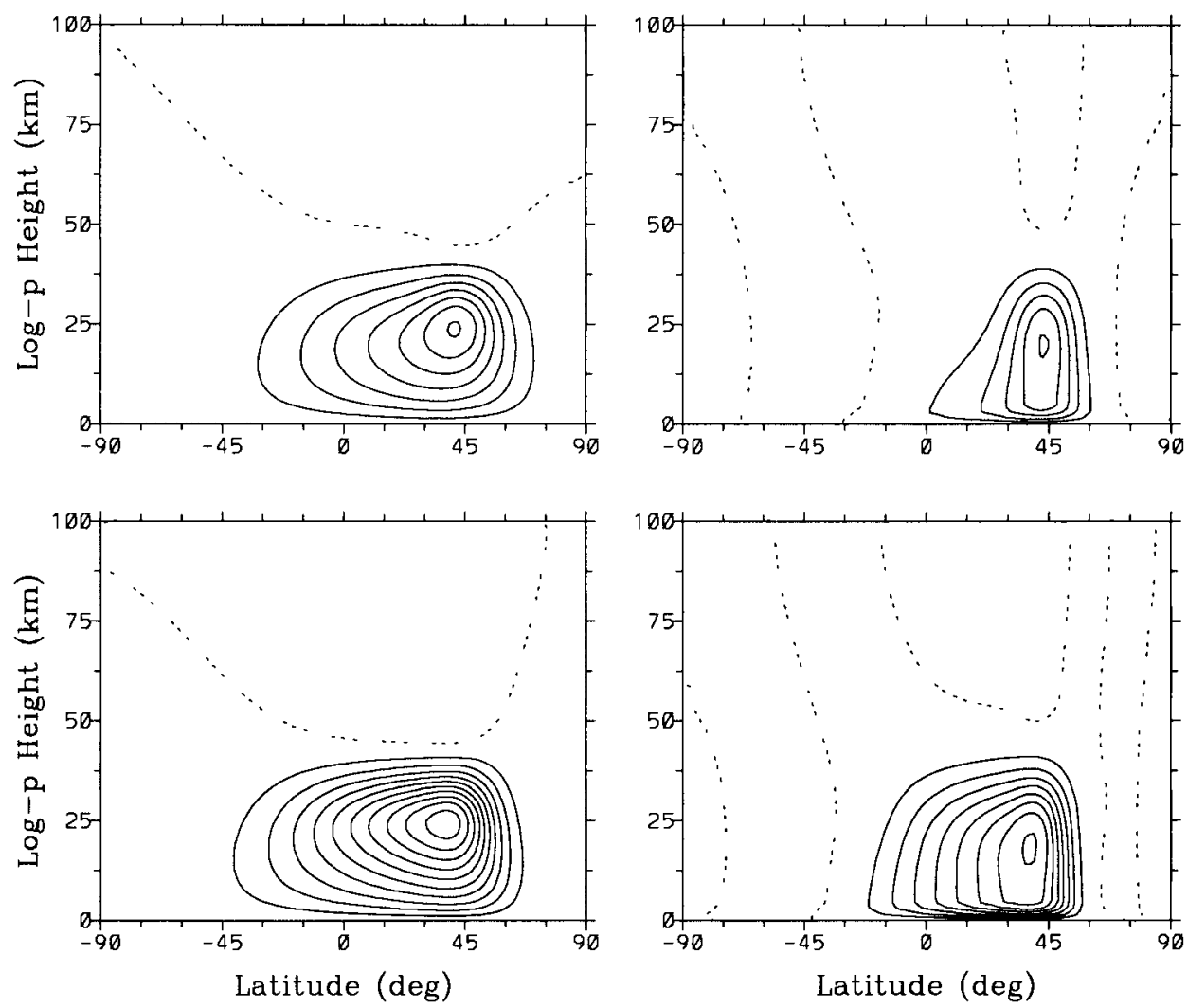

FIG. 17. Mass streamfunction for the residual circulation of the linearized balance model for a localized subtropical forcing at $45^{\circ} \mathrm{N}$ and $\alpha=1 / 10 \mathrm{day}^{-1}$. Panels on the left show the instantaneous response, panels on the right show the state after 100 days. The two pairs of panels correspond to the different $\bar{m}$ profiles given in Fig. 16. (Upper panels) Strong $\bar{m}_{\phi}$. (Lower panels) Homogenized $\bar{m}$. The contour interval for panels on the left is $0.75 \mathrm{~kg} \mathrm{~m}^{-1} \mathrm{~s}^{-1}$ and $2.5 \mathrm{~kg} \mathrm{~m}^{-1} \mathrm{~s}^{-1}$ for those on the right.

of $\psi$ and $a \Delta \phi$ to be its latitudinal scale, then in regions with $F \sim 0$ scaling analysis as in Holton et al. (1995) gives the relation

$$
\Delta z \sim \max \left[\frac{\cos \phi}{a} \sqrt{\frac{C}{A}} a \Delta \phi, \frac{\cos ^{2} \phi}{a^{2}} \frac{C}{A} \frac{(a \Delta \phi)^{2}}{H}\right],
$$

where the first relation applies when $\Delta z<H$ and the second when $\Delta z>H$. So in regions of weak $C$, such as in the regions with homogenized $\bar{m}$ (where $\partial M / \partial \phi$ is small or zero), the latitudinal scale of the streamfunction has to be broader for the vertical derivative term to be balanced by the latitudinal derivative term. It follows that the streamlines will extend farther from $\mathcal{F}$. In the case of overlap between $F$ and a parabolic region the two dominant terms are $A \partial^{2} \psi / \partial \phi^{2}$ and $F$ in the region of overlap. This also leads to a broadening of the streamfunction. The effect appears stronger than in the nonoverlapping case discussed above (Figs. 17 and 18) since the number of streamlines affected is much greater.

The change in the number of streamlines or, equivalently, the amplitude of $\psi$ in Fig. 19 (early time limit) comes from the $\bar{m} / a$ factor multiplying $\mathcal{F}$ in the expression for $F$. The basic-state $\bar{m}$ profiles used here have different values at the latitudes of the forcing, with the parabolic profile having a higher value. The increased meridional circulation demonstrates that the distribution of the zonal wind in the forcing region affects the streamfunction response through the forcing of the streamfunction equation and not just through its coefficients. However, the intensification of the streamfunction seen here has an unrealistic magnitude since the size of the parabolic region is exaggerated.

Since acceleration of the zonal wind by the diabatic circulation is not possible in regions of homogenized $\bar{m}$, only adiabatic heating and the thermal-wind relation enable the circulation to influence the zonal wind. The system can still evolve to steady state, but streamlines of the circulation are no longer forced to collapse onto $\bar{m}$ surfaces confined to the latitudes of the forcing region. Nonellipticity near the equator partly explains the rapidly decreasing rate of evolution to steady state and the breakdown of downward control in the Tropics. The central element of downward control is a feedback between the component of the circulation driven by the relaxational diabatic heating and the distribution of the heating itself. In regions of horizontally stratified $\bar{m}$, localized wave drag induces a diabatic heating quad- 

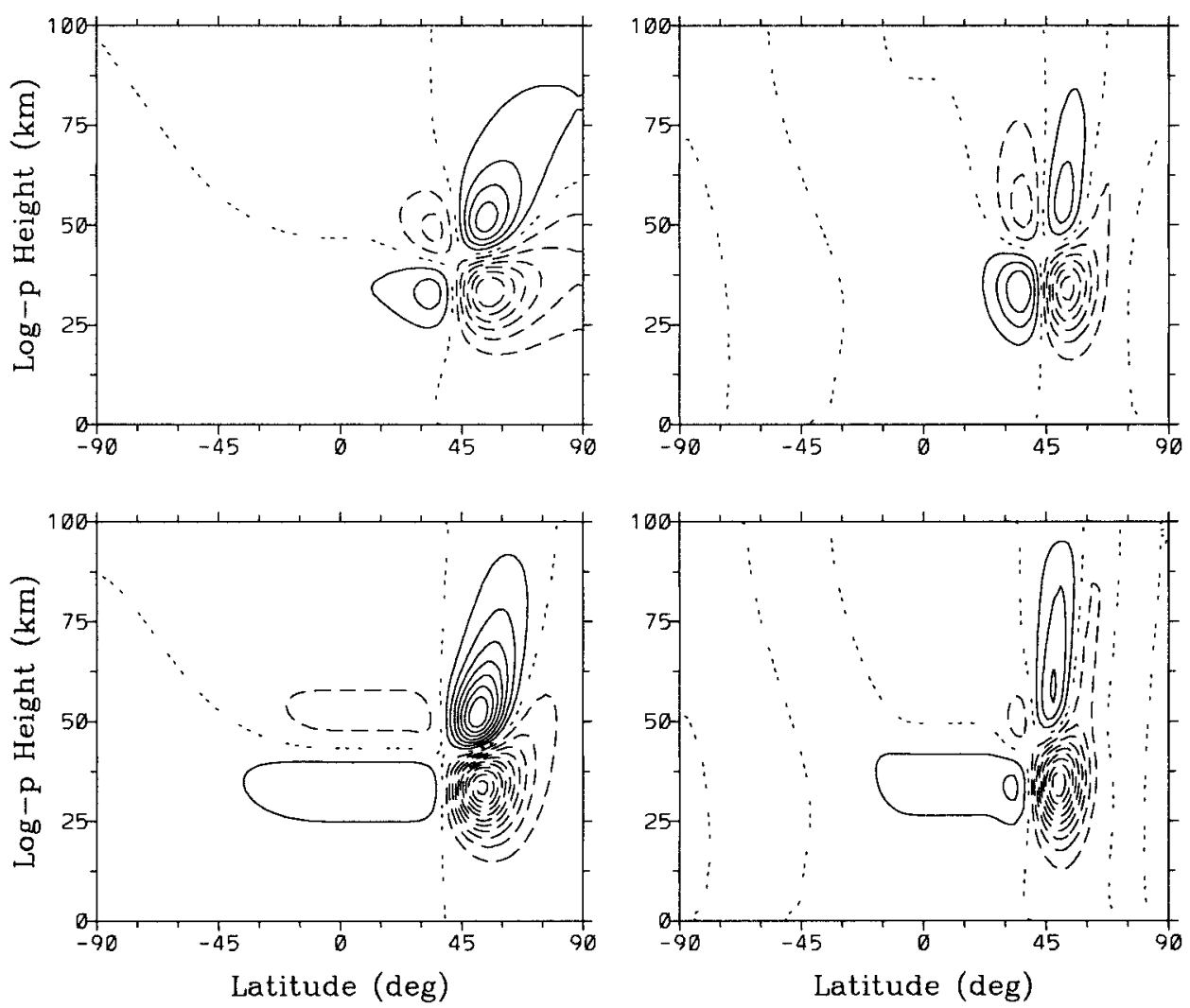

FIG. 18. Same as Fig. 17 but for the vertical velocity. The contour interval for panels on the left is $3 \times$ $10^{-5} \mathrm{~m} \mathrm{~s}^{-1}$ and $1 \times 10^{-4} \mathrm{~m} \mathrm{~s}^{-1}$ for those on the right.

rupole that drives a circulation of its own. This circulation adds to the wave-driven circulation component below $\mathcal{F}$ and diminishes it at other latitudes. In the Tropics, the diabatic heating induced circulation is much less effective in this role. Consequently, the essential feedback in the downward control effect breaks down.

The reasons for the different pattern of nonlocal diabatic heating response to low-latitude wave drag compared to the extratropics can be described in greater detail. Since streamlines of the wave-driven circulation are less concentrated below the levels of wave drag, the induced diabatic heating is more spread out, reflecting the $\bar{w}^{*}$ field. As a result, the circulation driven by diabatic heating on the tropical side is not effective in shifting the total circulation toward the region below the wave drag. Another difference at low latitudes is that changes in $\bar{u}$ have a smaller associated $\bar{T}$ response on account of the diminishing Coriolis factor in the thermal-wind relation. Nonlocal acceleration of the zonal wind through the Coriolis term $f \bar{v}^{*}$ also diminishes at low latitudes (unlike the local acceleration in the forcing region, which increases as the force is moved to lower latitudes). Consequently, the wave-driven circulation is less effective at inducing diabatic heating in the Tropics.

The results of this section show that the formation of weak $\partial \bar{m} / \partial \phi$ regions that extend away from the equator affects the diabatic circulation pattern induced by subtropical wave drag. Not only is there a deeper penetration of streamlines into the Tropics, but there is an enhancement of the circulation and upwelling as well. For background states with weak ellipticity a broadening of the tropical boundary layer of Plumb and Eluszkiewicz (1999) can be expected, since the tropical boundary layer is associated with the nonellipticity of the linear system at the equator in addition to viscosity. If significant homogenization of $\bar{m}$ in the real atmosphere were to occur, then the wave-driven circulation component of tropical upwelling would be boosted. Weak $\partial \bar{m} / \partial \phi$ does indeed occur near the tropical stratopause region possibly making this region more sensitive to subtropical wave drag (and leading, perhaps, to an enhanced easterly phase of the semiannual oscillation). The effect described by TK is likely a manifestation of this nonelliptic behavior.

\section{Tropical upwelling in a middle-atmosphere GCM}

The idealized model analysis is only an initial step in the study of the tropical upwelling problem. In this section we consider how much of the tropical upwelling in a realistic model of the atmosphere can be attributed to the Hadley circulation. CMAM is used for this pur- 

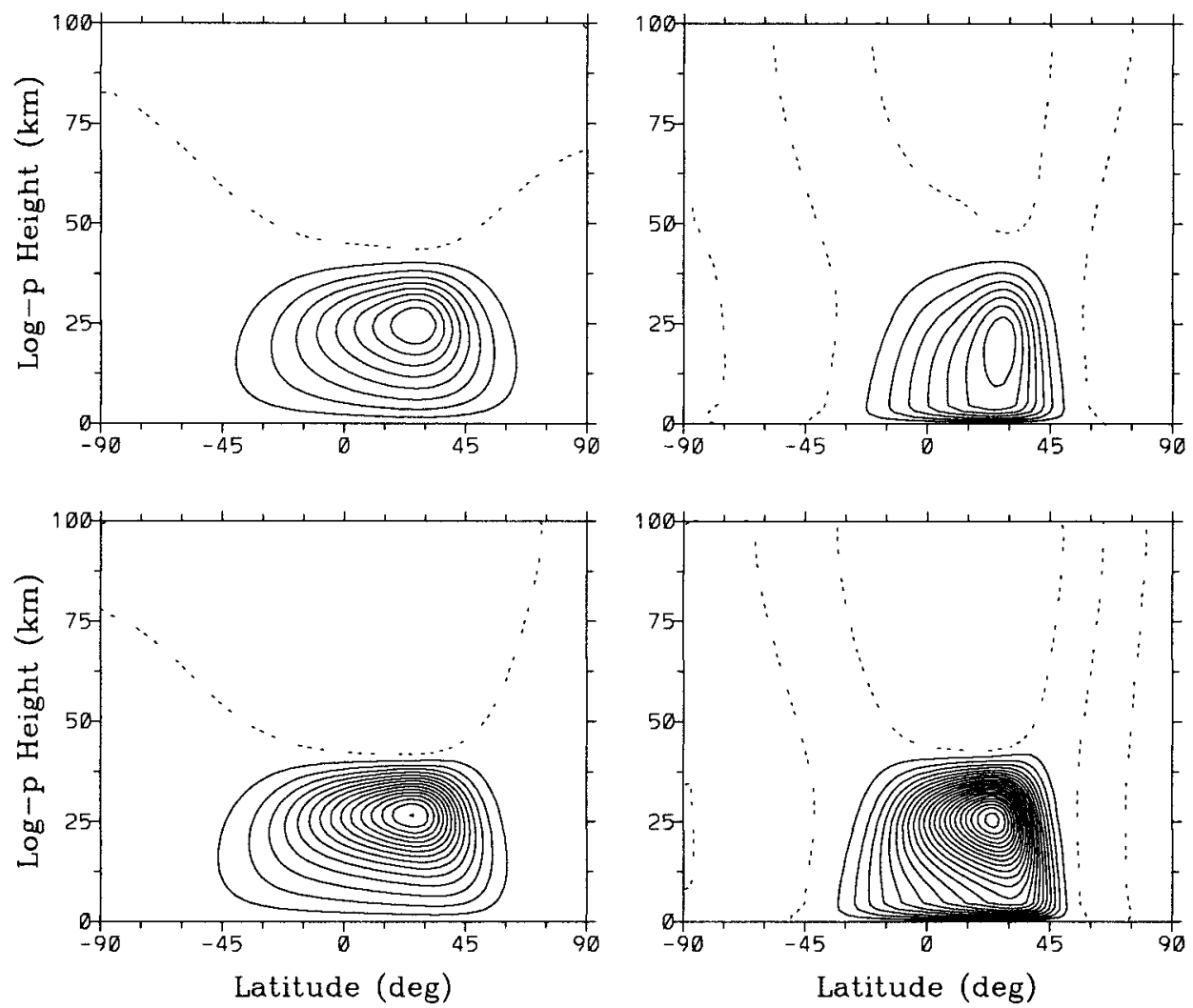

FIG. 19. Same as Fig. 17 but for forcing at $30^{\circ} \mathrm{N}$.

pose. This middle-atmosphere GCM extends from the surface to about $96-\mathrm{km}$ altitude (see Beagley et al. 1997). The annual mean vertical profiles of upwelling on the equator in CMAM and in the balance model simulation of SS (without mechanical forcing) using the comprehensive radiative transfer scheme are given in Fig. 21. The quantitative agreement in the upper stratosphere and stratopause region suggests that a large part of the CMAM upwelling originates from streamline and $\bar{m}$ contour mobility similar to that of the Hadley circulation near the equator. The CMAM annual mean upwelling in the middle and lower stratosphere, in contrast, is not captured by the balance model and is presumably dominated by the wave-driven component.

The increasing deformation of $\bar{m}$ isopleths with altitude (as is apparent from the CMAM $\bar{m}$ field, Fig. 13; see also SS Fig. 19) implies that the weakly nonlinear regime ceases to be valid in the tropical upper stratosphere. The nature of the mobility of $\bar{m}$ contours in this region is such that the Hadley circulation acts in concert with the wave-driven circulation to produce annual mean upwelling near the equator. Near the equator, the streamline pattern of the wave-driven circulation produced by the observed wave-drag distribution [e.g., based on Met Office (UKMO) assimilated data, see Rosenlof (1995), or from CMAM, see Fig. 22] has a similarity to that of the Hadley circulation at these altitudes. In both cases streamlines slant sideways-downward from the winter hemisphere into the summer hemisphere with an increasing degree of flatness (i.e., increasing $\bar{v}^{*}$ ) approaching the stratopause.

Further indication of the contribution of the Hadley circulation is evident in the seasonal evolution of the $\bar{w}^{*}$ field of CMAM at the 40-km level (Fig. 23, top panel), which is similar to the pattern of upwelling deduced from a radiative calculation using UKMO assimilated temperature data (cf. Fig. 10 of Randel et al. 1998). The maximum upwelling meanders from one side of the equator to the other at roughly comparable latitudes and the upwelling produced by the Hadley circulation in the balance model has a magnitude about half that of CMAM in the equatorial regions (Fig. 23, bottom panel). Figure 24 shows the upwelling pattern at the $23-\mathrm{km}$ level. Compared to the pattern at the $40-$ $\mathrm{km}$ level, the balance model upwelling has a much smaller magnitude and maximizes about 2 months earlier (during equinox seasons) and at higher latitudes, which is consistent with the behavior seen in CMAM. This equinoctial upwelling in the balance model is dominated by the transient associated with the rapid change in $\bar{T}_{\mathrm{rad}}$ rather than by the equinoctial Hadley circulation. However, there is a persistent equatorial upwelling in CMAM that is not represented in the balance model (cf. Fig. 21), which shifts the zero contour into the winter hemisphere during solstice seasons. Nevertheless, it appears from Fig. 24 that the seasonally evolving Hadley 

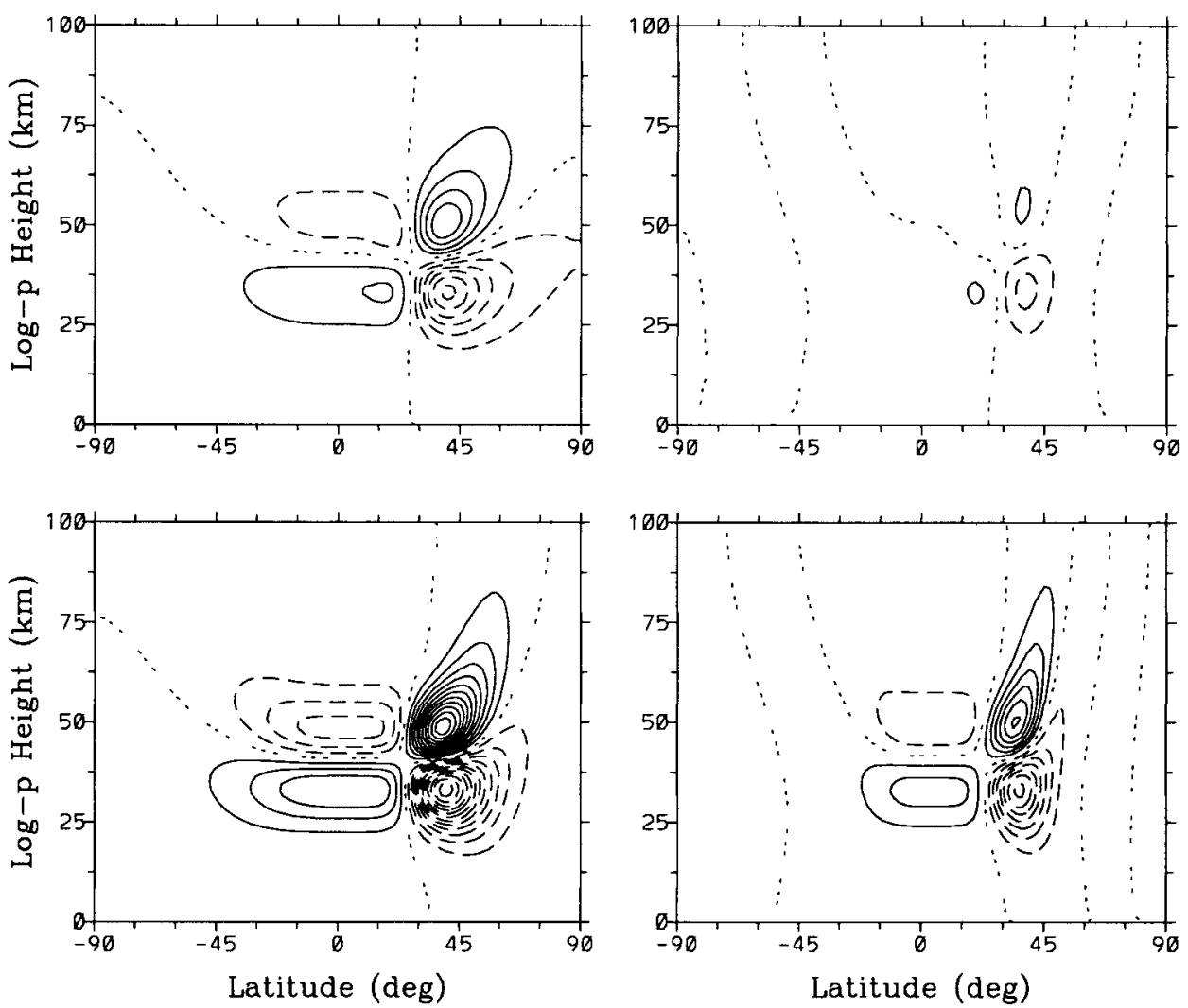

FIG. 20. Same as Fig. 18 but for forcing at $30^{\circ} \mathrm{N}$. The contour interval for panels on the left is $3 \times 10^{-5}$ $\mathrm{m} \mathrm{s}^{-1}$ and $3 \times 10^{-4} \mathrm{~m} \mathrm{~s}^{-1}$ for those on the right.

circulation could explain about $25 \%$ of the lower-stratospheric upwelling during solstice seasons in CMAM.

Since the TEM system is a good approximation to the zonal mean state of the primitive equations, which is close to being in balance even in the Tropics, there is reason to believe that to leading order, features of the Hadley circulation are simply superimposed on the wave-driven circulation (see SS, section 4). This is due to the absence of self-advection of the circulation in the balance system. As the results of SS indicate, such a streamline pattern produces a deflection of $\bar{m}$ isopleths into the winter hemisphere (as can be inferred from observations, Fig. 13; see also SS Fig. 6), which in turn deforms the streamfunction. It appears that this Hadleylike behavior of the full streamfunction near the equator can account for most of the observed annual mean upwelling at very low latitudes in the upper stratosphere. But away from the equator and in the lower stratosphere some other process is required.

Possible mechanisms by which annual mean upwelling is produced in the lower tropical stratosphere were the focus of section 3. Plumb and Eluszkiewicz (1999) have proposed one possibility, namely, the wave-driven circulation originating from higher latitudes together with weak relaxational damping. However, there appears to be some deformation of $\bar{m}$ in this region, which is consistent with advection of weak easterlies from the summer hemisphere (although not to the degree seen in the upper stratosphere), as discussed previously. This suggests that part of the annual mean upwelling may be explainable by the weakly nonlinear mechanism considered in section 4 .

\section{Conclusions}

The results presented here imply that the Hadley circulation makes a significant contribution to annual mean tropical upwelling only in the upper stratosphere. At these levels it can explain a large part of the annual mean upwelling seen in the CMAM GCM near the equator. However, the role of wave drag in the Tropics remains important at all levels and particularly in the lower stratosphere.

Wave drag, tropical and subtropical, is required to produce annual mean upwelling in the lower stratosphere. As seen in section 4, there exists a nonlinear mechanism that may explain part of the upwelling. Appeal to viscosity is not necessary when wave drag is confined away from the equator. However, the observed amplitude of the upwelling suggests that wave drag must penetrate quite close to the equator. The scenario considered here had wave drag distributed only in the winter hemisphere. In reality there is summer hemisphere wave 


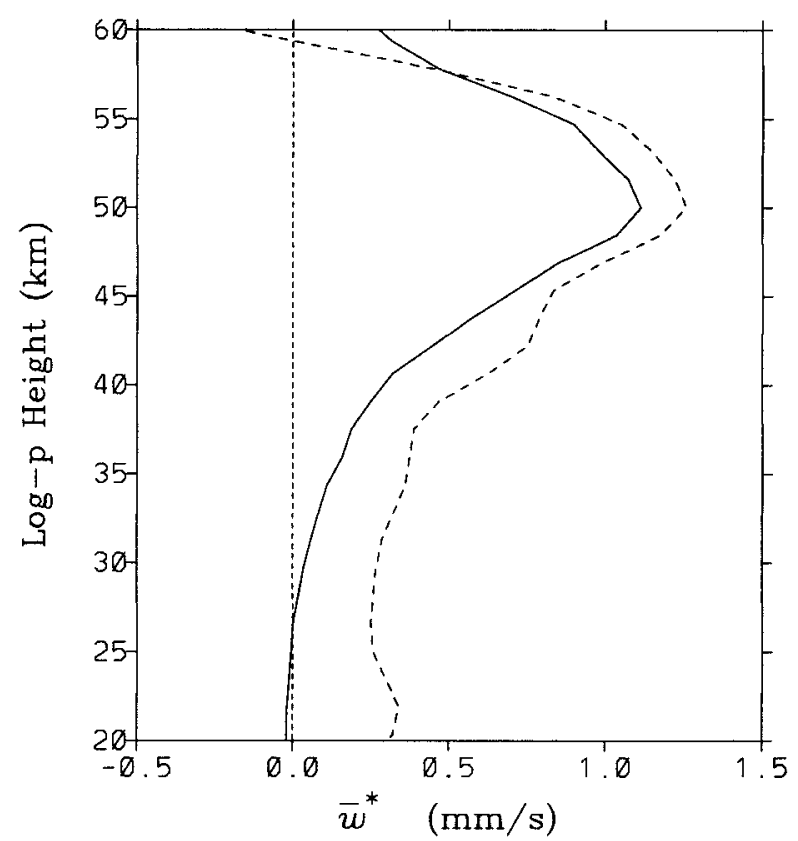

FIG. 21. Annual mean $\bar{w}^{*}$ on the equator for the nonlinear balance model without mechanical forcing (solid) and for CMAM (dash).

drag extending as high as $24 \mathrm{~km}$, as indicated by CMAM and other GCMs.

The inviscid nonlinear upwelling mechanism involves an asymmetric evolution in the force-induced diabatic heating compared to the linear case. In the nonlinear case, the induced diabatic heating decays more rapidly and fails to produce enough downwelling to cancel the force-driven upwelling during the declining phase of the forcing and afterward. Increased upwelling amplitude in the course of the seasonal cycle can thus translate into increased annual mean upwelling through greater nonlinearity.

In the lower tropical stratosphere, the linear viscous model becomes more relevant if weak wave drag extends across the Tropics. This is due to the fact that under the weakly nonlinear conditions in this region, small amplitude wave drag tends to stabilize $\bar{m}$ isopleths. In the absence of wave drag, the zonal mean model with climatological $\bar{T}_{\text {rad }}$ develops inertial adjustment in a narrow band near the equator, so it is possible that inertial instability contributes to the formation of a viscous equatorial boundary layer in the real atmosphere.

It appears that the Hadley circulation can partly explain the summer hemisphere location of the upwelling maximum during the seasonal cycle. During solstice seasons the balance model Hadley circulation gives an upwelling of about $0.1 \mathrm{~mm} \mathrm{~s}^{-1}$ at $30^{\circ}$ in the summer hemisphere at $23-\mathrm{km}$ altitude. In the opposite hemisphere there is a comparable level of downwelling and nearly zero vertical flow at the equator. However, the upwelling inferred from observations (see Fig. 2 of Plumb and Eluszkiewicz 1999) and from CMAM (Fig.
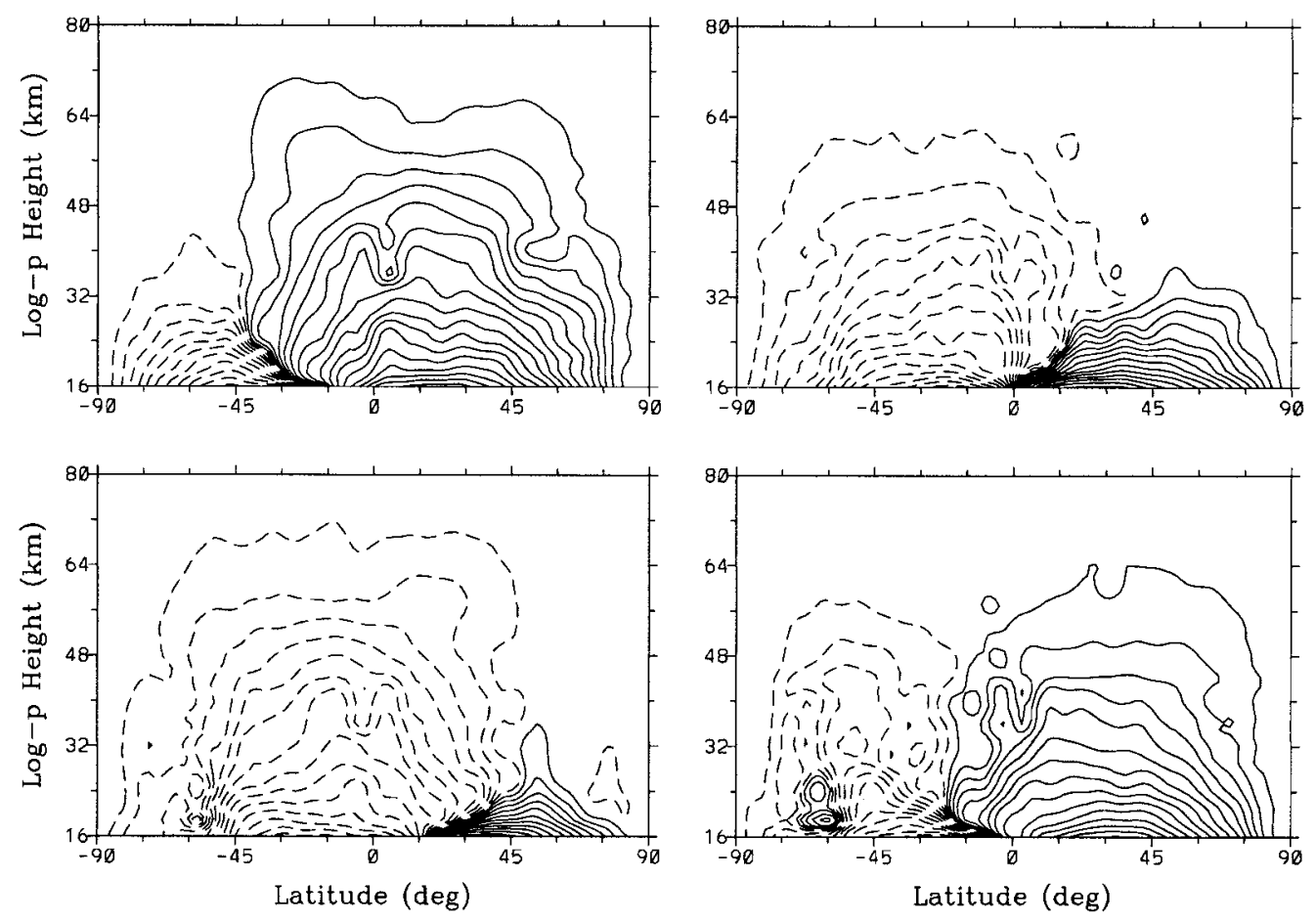

FIG. 22. Monthly mean CMAM residual mass streamfunction for Jan (upper left), Apr (upper right), Jul (lower left) and Oct (lower right). Contour level values are given by $40 x \exp (3.5|x|) \mathrm{kg} \mathrm{m}^{-1} \mathrm{~s}^{-1}$, where $x$ takes 48 uniformly spaced values in $-1 \leq x \leq 1$. 

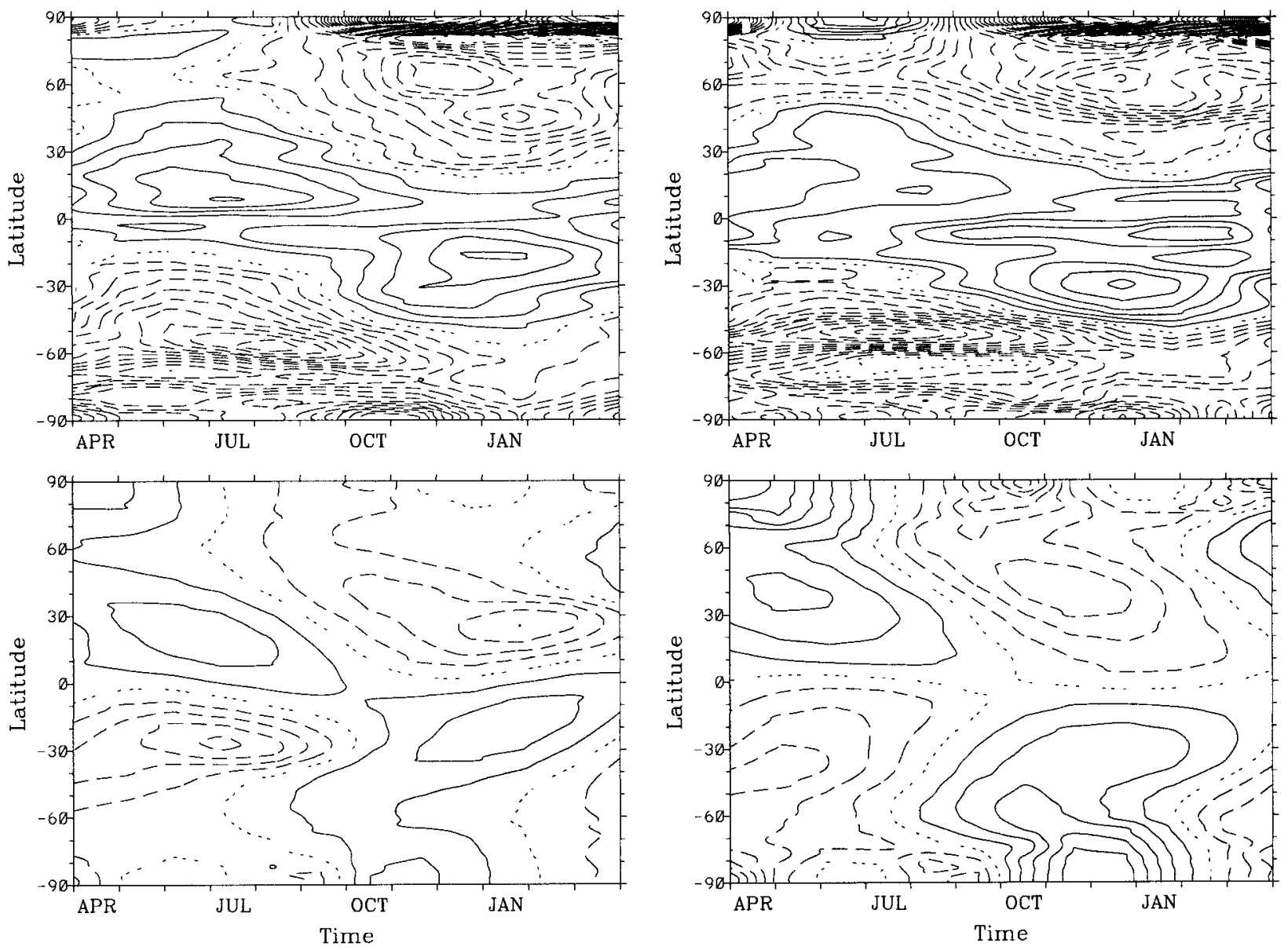

FIG. 23. Filtered time-latitude section of the monthly mean TEM vertical velocity on the $40-\mathrm{km}$ surface in CMAM (top panel) and the balance model without mechanical forcing (bottom panel). Contour interval is $3 \times 10^{-4} \mathrm{~m} \mathrm{~s}^{-1}$ for both panels.

24) suggests that an additional process is required to explain the strength of the seasonal upwelling maximum. At the equator itself the Hadley circulation is unable to produce significant upwelling in the lower stratosphere at any time of the year.

The effect of a reduced meridional $\bar{m}$ gradient on the wave-driven circulation considered in section 5 is likely important for low-latitude wave drag in the upper stratosphere and possibly near the equator in the vicinity of inertial instability. The parabolicity of the streamfunction operator near the region of highly deformed $\bar{m}$ contours close to the stratopause increases the cross-equatorial flow and upwelling. It could be said that the processes associated with the Hadley circulation condition the zonal mean tropical state to be more sensitive to subtropical wave drag. Some of the resulting enhanced upwelling could reach lower levels but decaying exponentially, $e$-folding at most over one density scale height. In the lower stratosphere both the observationally inferred and CMAM $\bar{m}$ distributions have a region of weak meridional gradient on the winter side of the

FIG. 24. Same as Fig. 23 but for the $23-\mathrm{km}$ surface. Contour interval is $1 \times 10^{-4} \mathrm{~m} \mathrm{~s}^{-1}$ for the top panel and $5 \times 10^{-5} \mathrm{~m} \mathrm{~s}^{-1}$ for the bottom panel.

equator. This can be expected to enhance the magnitude of the seasonal tropical upwelling, although not as significantly as in the upper stratosphere.

Another mechanism for the formation of weak $\bar{m}$ gradients in the Tropics, not considered here, is the QBO. During the easterly phase of the QBO there may be significant formation of near-parabolic regions at mid to lower levels of the tropical stratosphere. This has the potential to enhance tropical upwelling at the corresponding altitudes. However, the easterly phase of the QBO is also associated with a greater degree of tropical confinement from planetary waves, which could offset part or all of the upwelling enhancement.

Acknowledgments. We thank T. J. Dunkerton and R. A. Plumb for their comments. This work represents part of KS's Ph.D. thesis at the University of Toronto. He wishes to acknowledge support from an Ontario Graduate Scholarship, the Canadian MAM project, and the university. TGS is supported by the Natural Sciences and Engineering Research Council and the Meteorological Service of Canada. 


\section{REFERENCES}

Andrews, D. G., J. R. Holton, and C. B. Leovy, 1987: Middle Atmosphere Dynamics. Academic Press, 489 pp.

Beagley, S. R., J. de Grandpré, J. N. Koshyk, N. A. McFarlane, and T. G. Shepherd, 1997: Radiative-dynamical climatology of the first-generation Canadian middle atmosphere model. Atmos.Ocean, 35, 293-331.

Dunkerton, T. J., 1989: Nonlinear Hadley circulation driven by asymmetric differential heating. J. Atmos. Sci., 46, 956-974.

_ 1991: Nonlinear propagation of zonal winds in an atmosphere with Newtonian cooling and equatorial wavedriving. J. Atmos. Sci., 48, 236-263.

- 1997: The role of gravity waves in the quasi-biennial oscillation. J. Geophys. Res., 102, 26 053-26076.

Flemming, E. L., S. Chandra, J. J. Barnett, and M. Corney, 1990: Zonal mean temperature, pressure, zonal wind, and geopotential height as functions of latitude. Adv. Space Res., 10 (12), 11-59.

Grant, W. B., E. V. Browell, C. S. Longs, L. L. Stowe, R. G. Grainger, and A. Lambert, 1996: Use of volcanic aerosols to study the tropical stratospheric reservoir. J. Geophys. Res., 101, 39733988.

Haynes, P. H., C. J. Marks, M. E. McIntyre, T. G. Shepherd, and K. P. Shine, 1991: On the "downward control" of extratropical diabatic circulations by eddy-induced mean zonal forces. J. Atmos. Sci., 48, 651-678.

Held, I. M., and A. Y. Hou, 1980: Nonlinear axially symmetric circulations in a nearly inviscid atmosphere. J. Atmos. Sci., 37, $515-533$.

Holton, J. R., P. H. Haynes, M. E. McIntyre, A. R. Douglass, R. B. Rood, and L. Pfister, 1995: Stratosphere-troposphere exchange. Rev. Geophys., 33, 403-439.

Mote, P. W., and Coauthors, 1996: An atmospheric tape recorder: The imprint of tropical tropopause temperature on stratospheric water vapor. J. Geophys. Res., 101, 3989-4006.

_ - T. J. Dunkerton, M. E. McIntyre, E. A. Ray, P. H. Haynes, and J. M. Russell III, 1998: Vertical velocity, vertical diffusion, and dilution by midlatitude air in the tropical lower stratosphere. $J$. Geophys. Res., 103, 8651-8666.

Plumb, R. A., 1996: A "tropical pipe" model of stratospheric transport. J. Geophys. Res., 101, 3957-3972.

_ - and J. Eluszkiewicz, 1999: The Brewer-Dobson circulation: Dynamics of the tropical upwelling. J. Atmos. Sci., 56, 868-890.

Randel, W. J., F. Wu, J. M. Russell III, A. Roche, and J. W. Waters, 1998: Seasonal cycles and QBO variations in stratospheric $\mathrm{CH}_{4}$ and $\mathrm{H}_{2} \mathrm{O}$ observed in UARS HALOE data. J. Atmos. Sci., 55, 163-185.

Rosenlof, K. H., 1995: Seasonal cycle of the residual mean meridional circulation in the stratosphere. J. Geophys. Res., 100, 51735191.

Sankey, D., 1998: Dynamics of upwelling in the equatorial lower stratosphere. Ph.D. thesis, University of Cambridge, $232 \mathrm{pp}$.

Semeniuk, K., and T. G. Shepherd, 2001: The middle-atmosphere Hadley circulation and equatorial inertial adjustment. J. Atmos. Sci., 58, 3077-3096.

Shepherd, T. G., K. Semeniuk, and J. N. Koshyk, 1996: Sponge layer feedbacks in middle-atmosphere models. J. Geophys. Res., 101, 23 447-23 464.

Tung, K.-K., and J. S. Kinnersley, 2001: Mechanisms by which extratropical wave forcing in the winter stratosphere induces upwelling in the summer hemisphere. J. Geophys. Res., in press.

Yulaeva, E., J. R. Holton, and J. M. Wallace, 1994: On the cause of the annual cycle in tropical lower-stratospheric temperatures. $J$. Atmos. Sci., 51, 169-174. 